\title{
Topological Superconductivity in an Extended $s$-Wave Superconductor and Its Implication to Iron-Based Superconductors
}

\author{
Shengshan Qin, ${ }^{1,2}$ Chen Fang, ${ }^{3,4,1, *}$ Fu-Chun Zhang, ${ }^{1,2}$ and Jiangping $\mathrm{Hu}^{3,1,2, \dagger}$ \\ ${ }^{1}$ Kavli Institute of Theoretical Sciences, University of Chinese Academy of Sciences, \\ Beijing, 100049, China \\ ${ }^{2}$ CAS Center for Excellence in Topological Quantum Computation, \\ University of Chinese Academy of Sciences, Beijing, 100049, China \\ ${ }^{3}$ Beijing National Research Center for Condensed Matter Physics, and Institute of Physics, \\ Chinese Academy of Sciences, Beijing 100190, China \\ ${ }^{4}$ Songshan Lake Materials Laboratory, Dongguan, Guangdong 523808, China
}

(Received 13 June 2021; revised 28 September 2021; accepted 8 December 2021; published 15 February 2022)

In the presence of both space and time reversal symmetries, an $s$-wave $A_{1 g}$ superconducting state is usually topologically trivial. Here, we demonstrate that an exception can take place in a type of nonsymmorphic lattice structure. We specify the demonstration in a time reversal invariant system with a centrosymmetric space group $P 4 / \mathrm{nmm}$, the symmetry that governs iron-based superconductors, by showing the existence of a second-order topological state protected by a mirror symmetry. The topological superconductivity is featured by $2 \mathcal{Z}$ degenerate Dirac cones on the (10) edge and $\mathcal{Z}$ pairs of Majorana modes at the intersection between the (11) and (11) edges. The topological invariance and Fermi surface criterion for the topological state are provided. Moreover, we point out that the previously proposed $s$-wave state in iron-based superconductors, which features a sign-changed superconducting order parameter between two electron pockets, is such a topological state. Thus, these results not only open a new route to pursue topological superconductivity, but also establish a measurable quantity to settle one long-lasting debate on the pairing nature of iron-based superconductors.

DOI: 10.1103/PhysRevX.12.011030

Subject Areas: Condensed Matter Physics

\section{INTRODUCTION}

Stimulated by potential application in fault-tolerant quantum computation, the search for topological superconductors [1-8] is a central topic in condensed matter physics. After decades of efforts, great progress has been made both theoretically [9-24] and experimentally [25-36]. In recent years, motivated by the study in topological insulators [37-47], more and more attention has been paid to the topological superconducting phases protected by crystalline symmetries, dubbed as topological crystalline superconductors. The crystalline symmetries play a dramatic role in classifying the topological superconductors. On one hand, the crystalline symmetries greatly enrich the classification. For example, in the presence of mirror symmetry [48-51], rotational

\footnotetext{
*cfang@iphy.ac.cn

jphu@iphy.ac.cn
}

Published by the American Physical Society under the terms of the Creative Commons Attribution 4.0 International license. Further distribution of this work must maintain attribution to the author(s) and the published article's title, journal citation, and DOI. symmetry [52-58], or glide mirror symmetry [59-61], many new topological superconducting states beyond the Altland-Zirnbauer classification $[62,63]$ have been identified. On the other hand, the crystalline symmetries have strict constraints on the values of the topological indices. For instance, if a class-DIII superconductor is centrosymmetric with the superconducting order belonging to a trivial irreducible representation, it can hardly carry any topological nontrivial property [64-66]. Thus, normally, abundant $s$-wave centrosymmetric superconductors cannot be topological superconductors.

In this study, we show that the $s$-wave superconducting states in centrosymmetric superconductors can carry nontrivial topology in the presence of additional nonsymmorphic symmetries, i.e., the glide mirror symmetry or the screw rotation symmetry. This exception stems from anomalous band degeneracies induced by the nonsymmorphic symmetries. We specify the study with the space group $P 4 / \mathrm{nmm}$ (\#.129), the nonsymmorphic symmetry group that governs iron-based superconductors. A second-order topological superconducting state in the $A_{1 g}$ pairing channel is constructed and is characterized by a $2 \mathcal{Z}$ winding number protected by the mirror symmetry. The state hosts $2 \mathcal{Z}$ degenerate Dirac cones on the edge and $\mathcal{Z}$ pairs of 
Majorana modes at the corner, where the corresponding mirror symmetry is preserved. We develop a Fermi surface criterion for such topological superconductors. The previously proposed $s$-wave state for iron-based superconductors $[67,68]$, which are characterized by a sign-changed superconducting order parameter between two electron pockets, belongs to this exceptional class of topological states. Thus, the theory also establishes a directly measurable quantity to reveal the pairing nature in iron-based superconductors.

In the following, we first introduce the centrosymmetric space group $P 4 / \mathrm{nmm}$ in the quasi-two-dimensional (2D) case and analyze the group structure by studying the commutation relation between different symmetry operations. Then, we demonstrate that in such systems, due to the nonsymmorphic property, in the reciprocal space on the Brillouin zone boundary the corresponding little group has a unique group structure, leading to anomalous band degeneracies which can never be realized in the point group condition. Based on the anomalous band degeneracies, we show that topological superconductivity can be realized in such systems even if the pairing order is $s$-wave $\left(A_{1 g}\right)$. We also provide a Fermi surface criterion for the development of topological superconductivity. To verify the theory, we carry out numerical simulations based on a toy model and analytic analysis based on the low-energy edge Hamiltonian. The study is also generalized to threedimensional (3D) systems. After establishing the theory, we show that the theory can be applied to the previously proposed extended $s$-wave state in iron-based superconductors $[67,68]$. In a similar spirit as previous studies $[17,69]$, we suggest that, by studying the topological physics in the iron-based superconductors, essential information on the pairing nature can be revealed.

\section{GROUP STRUCTURE OF P4/nmm}

We begin with a brief review of the structure of the space group $G=P 4 / \mathrm{nmm}$. We consider a quasi-2D lattice structure shown in Fig. 1(a), similar to monolayer ironbased superconductors. To describe the symmetry group, we adopt the Seitz operators $\{\alpha \mid \tau\}$, which act on the lattice in the way $\{\alpha \mid \tau\} \mathbf{r}=\alpha \mathbf{r}+\tau$. It is easy to check the Seitz operators have the following properties:

$$
\begin{gathered}
\{\alpha \mid \tau\}^{-1}=\left\{\alpha^{-1} \mid-\alpha^{-1} \tau\right\}, \\
\left\{\alpha_{1} \mid \tau_{1}\right\}\left\{\alpha_{2} \mid \tau_{2}\right\}=\left\{\alpha_{1} \alpha_{2} \mid \alpha_{1} \tau_{2}+\tau_{1}\right\},
\end{gathered}
$$

where $\alpha$ is a point group operation and $\tau$ is a spatial translation. Apparently, the translation symmetry in the space group can be denoted as $\left\{E \mid t_{1} \mathbf{a}_{\mathbf{1}}+t_{2} \mathbf{a}_{2}\right\}$, with the primitive lattice translations $\mathbf{a}_{\mathbf{i}}$ and integers $t_{i}$. The quotient group $G / T$, with $T$ being the translation group, is specified by 16 symmetry operations and is expressed instructively as [70] (a)
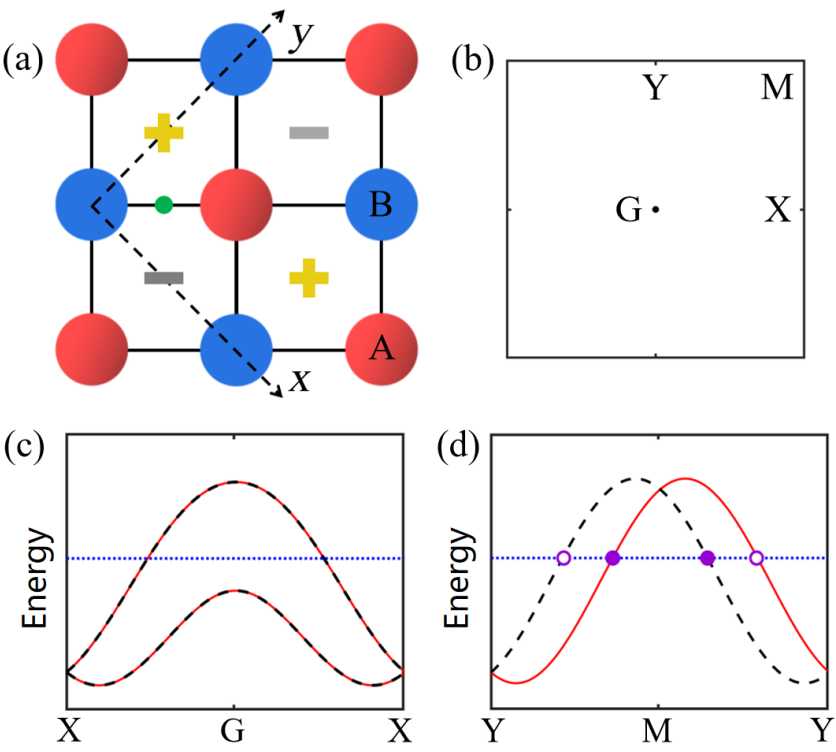

FIG. 1. (a) shows the monolayer case for the lattice structure respecting the space group $P 4 / \mathrm{nmm}$. The translational symmetry from sublattice $A$ to $B$ is broken by an intrinsic effective electric field normal to the $x y$ plane allowed by the symmetry [71-73], with \pm labeling the direction. The green point represents the inversion center. Throughout the paper, the [10] ([01]) direction is along the $x(y)$ axis. (b) shows the corresponding first Brillouin zone, with $G, X, M$, and $Y$ the $(0,0),(\pi, 0),(\pi, \pi)$, and $(0, \pi)$ points, respectively. Notice that $\left(k_{x}, k_{y}\right)$ in the Brillouin zone are defined according to $(x, y)$ in (a), and the lines $\Sigma_{Y}(M-Y-M)$ and $\Sigma_{G}(X-G-X)$ are invariant under the mirror symmetry $\left\{M_{y} \mid \mathbf{0}\right\}$. For a system respecting the space group $P 4 / \mathrm{nmm}$, its energy bands along $\Sigma_{G}$ and $\Sigma_{Y}$ can be sketched in (c) and (d), respectively, with the red lines representing the bands in the $\left\{M_{y} \mid \mathbf{0}\right\}$ invariant subspace with eigenvalue $m_{y}=+i$ and the black dashed lines the bands in the subspace with $m_{y}=-i$. In the $s$-wave state, according to the criterion in Eq. (8), whatever the pairing order $\Delta$ is like on $\Sigma_{G}$, it does not contribute to topological superconductivity, while the sign-changed pairing on $\Sigma_{Y}$ illustrated in (d) (open purple circles and closed purple dots represent opposite signs of $\Delta$ on the Fermi points) leads to second-order topological superconductivity protected by the mirror symmetry $\left\{M_{y} \mid \mathbf{0}\right\}$. The blue dotted lines in (c) and (d) label the Fermi level.

$$
G / T=D_{2 d} \otimes Z_{2},
$$

with $D_{2 d}$ the point group defined on the lattice sites and $Z_{2}$ a two-element group including the inversion symmetry defined at the center of the bond between two nearest lattice sites, as illustrated in Fig. 1(a). In Eq. (2), the quotient group is a direct product of the two subgroups in a sense that symmetry operations are equivalent if they differ by a lattice translation. According to Eq. (2), the quotient group $G / T$ can be generated by the generators of the two subgroups $D_{2 d}$ and $Z_{2}$ including $\left\{M_{y} \mid \mathbf{0}\right\},\left\{S_{4 z} \mid \mathbf{0}\right\}$, and $\left\{I \mid \tau_{0}\right\}$, with all the symmetry operations being defined at the lattice site in Fig. 1(a) and $\tau_{\mathbf{0}}=\mathbf{a}_{1} / 2+\mathbf{a}_{2} / 2$. The point 
group part of the above operations, i.e., $\alpha$ in the Seitz operator $\{\alpha \mid \tau\}$, acts on the coordinates in Fig. 1(a) as

$$
\begin{aligned}
M_{y}:(x, y, z) & \mapsto(x,-y, z), \\
S_{4 z}:(x, y, z) & \mapsto(y,-x,-z), \\
I:(x, y, z) & \mapsto(-x,-y,-z) .
\end{aligned}
$$

The other symmetry operations in $G / T$ are specified in the Appendix A.

Because of the special structure of the quotient group $G / T$ shown in Eq. (2), we can obtain the following two conclusions. (i) Since $D_{2 d}$ and $Z_{2}$ are two point groups defined on two inequivalent points, $G$ is nonsymmorphic and there are glide mirror and screw rotation operations in $G / T$. (ii) Different from the point group condition where the inversion symmetry always commutes with other point group symmetries, here the inversion symmetry in $Z_{2}$ does not commute with the point group symmetries in $D_{2 d}$; for example, $\left\{I \mid \tau_{\mathbf{0}}\right\}\left\{M_{y} \mid \mathbf{0}\right\}=\left\{E \mid \mathbf{a}_{\mathbf{2}}\right\}\left\{M_{y} \mid \mathbf{0}\right\}\left\{I \mid \tau_{\mathbf{0}}\right\}$.

\section{SYMMETRY CONSTRAINTS ON BLOCH STATES}

First, we analyze the constraint of the electronic structures by the nonsymmorphic symmetries on the boundaries of the Brillouin zone shown in Fig. 1(b), in time reversal invariant systems. We compare the $\Sigma_{Y}(M-Y-M)$ line with the $\Sigma_{G}(X-G-X)$ line in the Brillouin zone. The $\Sigma_{Y / G}$ line is invariant under the group $C_{2 v} \otimes Z_{2}$, which includes the following symmetry operations:

$$
\begin{array}{lrr}
\{E \mid \mathbf{0}\}, & \left\{C_{2 z} \mid \mathbf{0}\right\}, \quad\left\{I \mid \tau_{\mathbf{0}}\right\}, & \left\{M_{z} \mid \tau_{\mathbf{0}}\right\}, \\
\left\{M_{x} \mid \mathbf{0}\right\}, & \left\{M_{y} \mid \mathbf{0}\right\}, \quad\left\{C_{2 x} \mid \tau_{\mathbf{0}}\right\}, & \left\{C_{2 y} \mid \tau_{\mathbf{0}}\right\},
\end{array}
$$

where $\tau_{0}=\mathbf{a}_{1} / 2+\mathbf{a}_{2} / 2$ and all the symmetry operations are defined at the lattice site in Fig. 1(a). Besides the crystalline symmetries, we can also consider the operation $\left\{I \mid \tau_{\mathbf{0}}\right\} \Theta$ with $\Theta$ being the time reversal symmetry. $\left\{I \mid \tau_{\mathbf{0}}\right\} \Theta$ is antiunitary and leaves every $\mathbf{k}$ point unchanged in the Brillouin zone. We can use the above symmetries to classify the eigenstates on $\Sigma_{Y / G}$. Here, we focus on the mirror symmetry $\left\{M_{y} \mid \mathbf{0}\right\}$, which allows us to specify eigenvalues of Bloch states as $m_{y}= \pm i$. In the spinful condition, the symmetries satisfy

$$
\begin{aligned}
\left(\left\{I \mid \tau_{\mathbf{0}}\right\} \Theta\right)\left(\left\{I \mid \tau_{\mathbf{0}}\right\} \Theta\right)|\varphi(\mathbf{k})\rangle & =-|\varphi(\mathbf{k})\rangle, \\
\left(\left\{I \mid \tau_{\mathbf{0}}\right\} \Theta\right)\left\{M_{y} \mid \mathbf{0}\right\}|\varphi(\mathbf{k})\rangle & =e^{i k_{y}}\left\{M_{y} \mid \mathbf{0}\right\}\left(\left\{I \mid \tau_{\mathbf{0}}\right\} \Theta\right)|\varphi(\mathbf{k})\rangle
\end{aligned}
$$

Equation (5a) provides the Kramers' degeneracy which applies for every $\mathbf{k}$ point in the Brillouin zone. In deriving Eq. (5b), we utilize the following facts.
$\left\{I \mid \tau_{\mathbf{0}}\right\}\left\{M_{y} \mid \mathbf{0}\right\}=\left\{E \mid \mathbf{a}_{\mathbf{2}}\right\}\left\{M_{y} \mid \mathbf{0}\right\}\left\{I \mid \tau_{\mathbf{0}}\right\}$, and the time reversal symmetry commutes with the crystalline symmetries in a paramagnetic system. The phase factor in Eq. (5b) makes the commutation relation between the symmetries $\left\{I \mid \tau_{\mathbf{0}}\right\}$ and $\left\{I \mid \tau_{\mathbf{0}}\right\}\left\{M_{y} \mid \mathbf{0}\right\} \mathbf{k}$ dependent. Specifically, along the lines $\Sigma_{Y}$ and $\Sigma_{G}$, it gives

$$
\begin{aligned}
& k_{y}=\pi:\left[\left\{I \mid \tau_{\mathbf{0}}\right\} \Theta,\left\{M_{y} \mid \mathbf{0}\right\}\right]_{+}=0, \\
& k_{y}=0:\left[\left\{I \mid \tau_{\mathbf{0}}\right\} \Theta,\left\{M_{y} \mid \mathbf{0}\right\}\right]_{-}=0,
\end{aligned}
$$

where []$_{-}$and []$_{+}$label the commutation and anticommutation operations, respectively [74]. Based on Eqs. (5) and (6), for a Bloch state with eigenvalue $m_{y}$, there is a degenerate state $-m_{y}$ on $\Sigma_{G}$ and a degenerate state $m_{y}$ on $\Sigma_{Y}$.

At the $Y$ and $M$ points on $\Sigma_{Y}$, besides the above symmetries, the twofold rotational symmetry $\left\{C_{2 z} \mid \mathbf{0}\right\}$ also preserves. In the spinful condition, $\left\{C_{2 z} \mid \mathbf{0}\right\}$ always maps a state with the mirror eigenvalue $m_{y}$ to a state with $-m_{y}$. Therefore, the bands must be fourfold degenerate at $Y$ and $M$. The degeneracy between the $m_{y}$ and $-m_{y}$ states at $Y$ and $M$ is also consistent with the constraint of the time reversal symmetry. Based on the above analysis, we can obtain an overall picture on the bands along the $\Sigma_{Y / G}$ line as illustrated in Figs. 1(c) and 1(d). Notice that the bands at $X$ in Fig. 1(c) are also fourfold degenerate, because the $X$ point and the $Y$ point are related by the fourfold rotational symmetry in the space group.

\section{IV. $s$-WAVE $\left(A_{1 g}\right)$ TOPOLOGICAL SUPERCONDUCTIVITY}

We consider $s$-wave superconductivity in the 2D lattice in Fig. 1(a). Here, we focus on the time reversal invariant superconductors, which belong to class DIII and are characterized by a $\mathcal{Z}_{2}$ topological index according to the Altland-Zirnbauer classification [62,63]. The crystalline symmetries have strict constraints on the values of the topological index. In the presence of inversion symmetry, the above $\mathcal{Z}_{2}$ index in an $s$-wave superconductor is always trivial [64-66]. A brief proof is given in Appendix D. Though $\mathcal{Z}_{2}$ is trivial, as we show, the $s$-wave superconductors respecting the lattice in Fig. 1(a) can be mirror-symmetry-protected topological superconductors. Specifically, we consider the topological classification of $s$-wave superconducting states protected by the mirror symmetry $\left\{M_{y} \mid \mathbf{0}\right\}$ in the following.

In class-DIII superconductors, there always exists the chiral symmetry $\mathcal{C}$, which is the product of the time reversal symmetry and the particle-hole symmetry. In the $s$-wave state, the chiral symmetry commutates with the crystalline symmetries and is preserved in each of the $\left\{M_{y} \mid \mathbf{0}\right\}$ invariant subspaces $[48,50]$. Now we consider the two $\left\{M_{y} \mid \mathbf{0}\right\}$ invariant lines $\Sigma_{Y}$ and $\Sigma_{G}$, in the Brillouin zone, 
and treat them as two one-dimensional (1D) subsystems. The winding number in each of the mirror subspaces along the $\Sigma_{Y / G}$ line is given by [50]

$$
w_{Y / G}^{ \pm i}=\int_{Y / G} \frac{d k_{x}}{2 \pi} \operatorname{tr}\left[\mathcal{C H}_{ \pm i}^{-1}(\mathbf{k}) \partial_{k_{x}} \mathcal{H}_{ \pm i}(\mathbf{k})\right]
$$

where $\mathcal{H}_{ \pm i}(\mathbf{k})$ is the superconducting Hamiltonian in the $m_{y}= \pm i$ mirror subspace. The winding numbers along the two lines are dramatically different due to the different symmetry-enforced band degeneracies along the two lines shown in Figs. 1(c) and 1(d). Considering the time reversal and space inversion symmetries together with the constraints in Eq. (6), we can obtain the following conclusions (details in Appendix C): (i) On $\Sigma_{G}$, the inversion symmetry preserves in each mirror invariant subspace, leading to the winding number always being trivial, namely, $w_{G}^{+i}=$ $-w_{G}^{-i}=0$; (ii) on $\Sigma_{Y}$, the inversion symmetry no longer preserves in the mirror invariant subspaces, and the classification is $w_{Y}^{+i}=-w_{Y}^{-i}=2 \mathcal{Z}$. The results can be further understood by calculating the $1 \mathrm{D}$ winding numbers through the Fermi surface criterion $[64,69,75]$

$$
w=\frac{1}{2} \sum_{j} \operatorname{sgn}\left[v\left(k_{F, j}\right) \Delta\left(k_{F, j}\right)\right],
$$

where $v\left(k_{F, j}\right)$ and $\Delta\left(k_{F, j}\right)$ are the Fermi velocity and superconducting pairing at the $j$ th Fermi point, respectively, and sgn[] is the sign function. For instance, to calculate $w_{Y / G}^{+i}$, the summation in Eq. (8) includes all the Fermi points with mirror eigenvalue $m_{y}=+i$ on the $\Sigma_{Y / G}$ line, with $v\left(k_{F}\right)$ being the corresponding Fermi velocity along the $\Sigma_{Y / G}$ line and $\Delta\left(k_{F}\right)$ the superconducting pairing at the corresponding Fermi point; the winding number in the $m_{y}=-i$ mirror subspace can be calculated similarly. On the other hand, for an $s$-wave centrosymmetric superconducting state, the pairing order is an even function of $\mathbf{k}$. The Fermi velocity on $\Sigma_{G}$ is odd of $\mathbf{k}$ in each mirror subspace as indicated in Fig. 1(c), leading to $w_{G}^{ \pm i}=0$ according to the criterion in Eq. (8). However, on $\Sigma_{Y}$, the bands are no longer symmetric between $\mathbf{k}$ and $-\mathbf{k}$. The nonzero $w_{Y}^{ \pm i}$ can be generated if the pairing order has a sign change as indicated in Fig. 1(d). Specifically, the topological number is characterized by $\left|w_{Y}^{ \pm i}\right|$ Dirac cones degenerate at $k_{y}=\pi$ on the (10) edge. It is worth mentioning that the above analysis also applies for the mirror symmetry $\left\{M_{x} \mid \mathbf{0}\right\}$, since $\left\{M_{x} \mid \mathbf{0}\right\}$ and $\left\{M_{y} \mid \mathbf{0}\right\}$ are related by the fourfold rotational symmetry in the space group $P 4 / \mathrm{nmm}$.

We emphasize that the above topological superconductivity is unique for centrosymmetric superconductors governed by nonsymmorphic groups, since the anomalous band degeneracy in Fig. 1(d) can occur only in the presence of the nonsymmorphic symmetries.

\section{LATTICE MODEL}

To verify the above analysis, we construct a simple two-orbital $\left(p_{x}\right.$ and $p_{y}$ ) model in the lattice in Fig. 1(a). The $p_{x}\left(p_{y}\right)$ orbital is defined along the $x(y)$ direction in the lattice. The band structure with respect to the $P 4 / \mathrm{nmm}$ symmetry can be generally described by

$$
\begin{aligned}
\mathcal{H}_{0}= & t \cos k_{x} s_{0}\left(\sigma_{0}+\sigma_{3}\right) \eta_{0}+t \cos k_{y} s_{0}\left(\sigma_{0}-\sigma_{3}\right) \eta_{0} \\
& +4 t_{1} \cos \frac{k_{x}}{2} \cos \frac{k_{y}}{2} s_{0} \sigma_{0} \eta_{1}-4 t_{2} \sin \frac{k_{x}}{2} \sin \frac{k_{y}}{2} s_{0} \sigma_{1} \eta_{1} \\
& -\lambda_{R} \sin k_{x} s_{2}\left(\sigma_{0}+\sigma_{3}\right) \eta_{3}-\lambda_{R} \sin k_{y} s_{1}\left(\sigma_{0}-\sigma_{3}\right) \eta_{3} \\
& +\frac{\lambda}{2} s_{3} \sigma_{2} \eta_{0},
\end{aligned}
$$

where $s_{i}, \sigma_{i}$, and $\eta_{i}$ are the Pauli matrices standing for the spin, orbital, and sublattice degrees of freedom, respectively. In the model, $t$ is the nearest-neighbor $(\mathrm{NN})$ intrasublattice intraorbital hopping, $t_{1 / 2}$ is the $\mathrm{NN}$ intersublattice intraorbital or interorbital hopping, $\lambda$ is the atomic spin-orbit coupling, and $\lambda_{R}$ is the Rashba-type spinorbit coupling allowed by the symmetry group $P 4 / \mathrm{nmm}$. It takes the form $i \lambda_{R}(\mathbf{d} \times \mathbf{s}) \cdot \mathbf{e}_{\mathbf{z}}$ in real space, with $\mathbf{d}$ the intrasublattice $\mathrm{NN}$ vector and $\mathbf{e}_{\mathbf{z}}$ the direction of the effective electric field shown in Fig. 1(a). Notice that we preserve only the intraorbital $\lambda_{R}$, with the $\pi$-bond type omitted. We emphasize that the effective electric field inducing $\lambda_{R}$ is an intrinsic result of the symmetry group. Such inversion-symmetric polarization has been identified both theoretically and experimentally in previous studies [71-73,76]. In iron-based superconductors, this term stems from the noncoplanar cations and anions.

We set the parameters in Eq. (9) as $\left\{t, t_{1}, t_{2}, \lambda, \lambda_{R}\right\}=$ $\{-1.0,0.4,0.6,0.3,0.75\}$ and obtain the band structures in Fig. 2. Based on the lattice model, one can directly verify the different band degeneracies on $\Sigma_{G}$ and $\Sigma_{Y}$, by decoupling $\mathcal{H}_{0}$ according to the mirror symmetry $\left\{M_{y} \mid \mathbf{0}\right\}$ on these two lines (details in Appendix B).

An $s$-wave $\left(A_{1 g}\right)$ superconductivity is described generally by $\mathcal{H}_{\mathrm{sc}}=\left[\Delta_{0}+2 \Delta_{1}\left(\cos k_{x}+\cos k_{y}\right)\right] s_{0} \sigma_{0} \eta_{0}$ in the

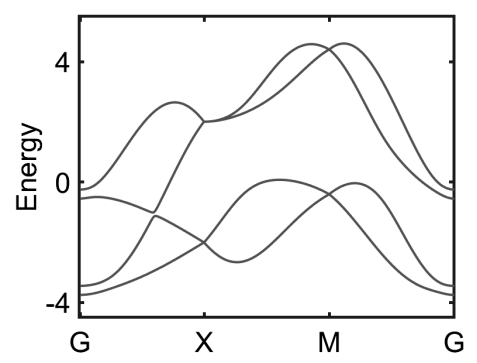

FIG. 2. The band structures correspond to the model Hamiltonian $\mathcal{H}_{0}$ in Eq. (9). $G, X$, and $M$ are the high-symmetry points shown in Fig. 1(b). The parameters are chosen as $\left\{t, t_{1}, t_{2}, \lambda, \lambda_{R}\right\}=\{-1.0,0.4,0.6,0.3,0.75\}$. 
basis $\psi^{\dagger}(\mathbf{k})=\left[c^{\dagger}(\mathbf{k}), i s_{2} c(-\mathbf{k})\right]$, where $\Delta_{0}$ is the on-site intraorbital pairing and $\Delta_{1}$ is the NN intrasublattice intraorbital pairing. The total superconducting Hamiltonian corresponding to the model in Eq. (9) takes the form

$$
\mathcal{H}_{\mathrm{BdG}}=\left(\mathcal{H}_{0}-\mu\right) \tau_{3}+\mathcal{H}_{\mathrm{sc}} \tau_{1}
$$

where the Pauli matrix $\tau_{i}$ is defined in Nambu space and $\mu$ is the chemical potential. The matrix forms of the related symmetry operations are listed as follows. The time reversal symmetry and the chiral symmetry take the form $\Theta=i s_{2} \sigma_{0} \eta_{0} \tau_{0} K$ and $\mathcal{C}=s_{0} \sigma_{0} \eta_{0} \tau_{2}$, respectively, with $K$ the complex conjugation operation, and the particlehole symmetry can be obtained as $\mathcal{P}=\mathcal{C} \Theta$; the three generators of the quotient group $G / T$ in Eq. (2) have the form $\mathcal{I}=e^{-i \mathbf{k} \cdot \tau_{0}} s_{0} \sigma_{0} \eta_{1} \tau_{0}, \quad \mathcal{M}_{y}=i s_{2} \sigma_{3} \eta_{0} \tau_{0}$, and $\mathcal{S}_{4 z}=i e^{i s_{3} \pi / 4} \sigma_{2} \eta_{0} \tau_{0}$, with $\mathcal{I}, \mathcal{M}_{y}$, and $\mathcal{S}_{4 z}$ corresponding to the symmetry operations $\left\{I \mid \tau_{\mathbf{0}}\right\},\left\{M_{y} \mid \mathbf{0}\right\}$, and $\left\{S_{4 z} \mid \mathbf{0}\right\}$, respectively.

To show the topological superconductivity protected by the mirror symmetry $\left\{M_{y} \mid \mathbf{0}\right\}$, we simulate the edge states on the boundary normal to mirror plane, i.e., the (10) edge. There are two different conditions in which the Fermi surfaces and pairing nodes are located around (i) the $M$ point and (ii) the $G$ point in the Brillouin zone, respectively, (a)
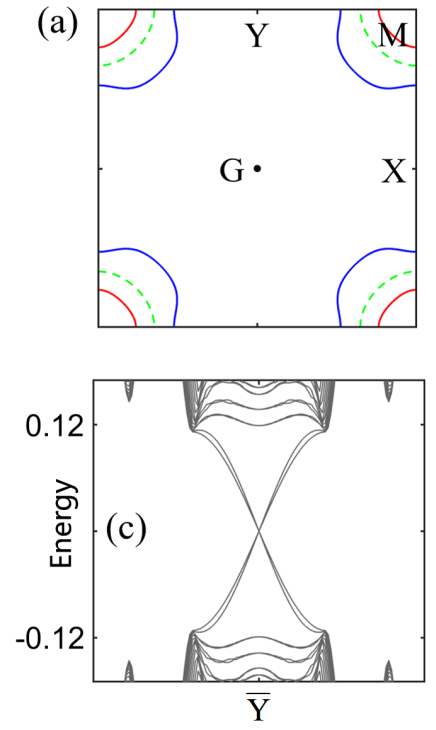

(b)
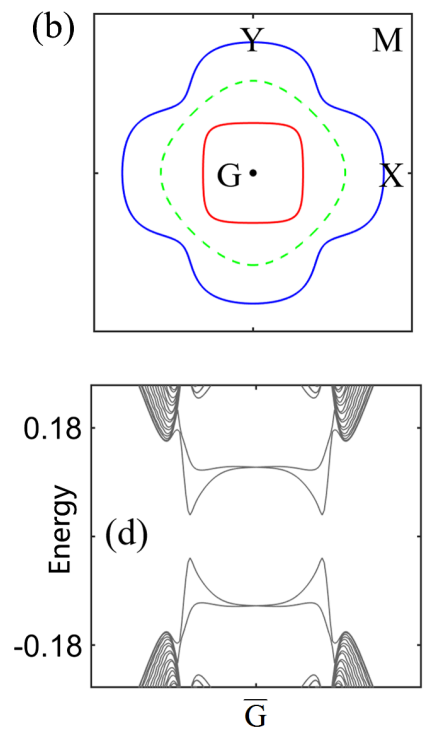

FIG. 3. (a) and (b) show the normal-state Fermi surfaces (solid lines) and the pairing nodes (dashed lines) for the Hamiltonian in Eq. (10). The red (blue) color in the figures means a positive (negative) superconducting pairing order on the Fermi surface. (c) and (d) show the edge modes on the (10) edge corresponding to the states depicted in (a) and (b), respectively. In (c) and (d), $\bar{Y}$ and $\bar{G}$ are the projection points of $Y$ and $G$ on the edge, respectively. The parameters are chosen as $\Delta_{0}=-0.58$, $\Delta_{1}=-0.2$, and the chemical potential $\mu=3.6$ for (a) and (c) and $\Delta_{0}=-0.3, \Delta_{1}=0.2$, and $\mu=0.8$ for (b) and (d). The other parameters are the same as those in Fig. 2. as shown in Figs. 3(a) and 3(b). In the first case, there are two Dirac cones degenerate at $k_{y}=\pi$ on the boundary, shown in Fig. 3(c), indicating $\left|w_{Y}^{ \pm i}\right|=2$. In the second case, there is no gapless mode as shown in Fig. 3(d), indicating a topological trivial state. To see that the results are consistent with the Fermi surface criterion in Eq. (8), we draw the Fermi surfaces and the vanishing lines of the superconducting orders, shown in Figs. 3(a) and 3(b). It is worth mentioning that on the (01) edge the results are similar, and the gapless edge modes corresponding to the state in Fig. 3(a) are protected by the mirror symmetry $\left\{M_{x} \mid \mathbf{0}\right\}$.

\section{MAJORANA CORNER MODES}

In fact, the mirror-protected topological superconductivity is a second-order topological state [77-83]. It supports Majorana modes at the corner with a $\mathcal{Z}$ classification equal to $\left|w_{Y}^{ \pm i}\right| / 2$. To verify this, we first simulate the edge states on the (11) edge under the same Fermi surface condition in Fig. 3(a). The (11) edge has neither $\left\{M_{y} \mid \mathbf{0}\right\}$ nor $\left\{M_{x} \mid \mathbf{0}\right\}$ symmetry. As expected, as shown in Fig. 4(a), no gapless modes survive, because $\left\{M_{y} \mid \mathbf{0}\right\}$ and $\left\{M_{x} \mid \mathbf{0}\right\}$ are no longer maintained. Furthermore, we simulate the corner modes localized at the intersection between the (11) and (11) edges. As shown in Fig. 4(b), at each corner there exists one pair of zero-energy modes, indicating secondorder topological superconductivity.

The rise of the corner Majorana modes is guaranteed by the mirror symmetries $\left\{M_{y} \mid \mathbf{0}\right\}$ and $\left\{M_{x} \mid \mathbf{0}\right\}$. This can be clearly verified by considering the effective theory on the edges, as sketched in Figs. 4(c) and 4(d). We first consider the $\left\{M_{y} \mid \mathbf{0}\right\}$ symmetry. We start with the gapless modes on the (10) edge illustrated in Fig. 4(c), corresponding to the $\left|w_{Y}^{ \pm i}\right|=2$ result shown in Fig. 3(c). On this edge, there are the time reversal symmetry, the particle-hole symmetry, the chiral symmetry, and the mirror symmetry. With the constraints of these symmetries and a proper gauge choice, the effective theory can be written as $\mathcal{H}_{\text {eff }}=v k_{y} s_{3} \kappa_{3}$, with $v$ the Fermi velocity, $s_{i}$ the Pauli matrices labeling the spin degree of freedom, and $\kappa_{i}$ the Pauli matrices for the remaining degree for the edge Dirac cones (details in Appendix E).

Now we gradually bend edge (10) into a right angle, with the two sides along the [11] and [11 $]$ directions. In this procedure, the mirror symmetry $\left\{M_{y} \mid \mathbf{0}\right\}$ is preserved, mapping the (11) edge to the (11) edge. However, the gapless modes on each edge gain a mass and are gapped out, as the mirror symmetry is not maintained on the (11) or (11) edge. The gapped modes on these two edges take the form (details in Appendix E)

$$
\begin{aligned}
& \mathcal{H}_{\text {eff }}^{(11)}=v k s_{3} \kappa_{3}+m_{(11)} s_{0} \kappa_{1}, \\
& \mathcal{H}_{\text {eff }}^{(1 \overline{1})}=v k s_{3} \kappa_{3}+m_{(1 \overline{1})} s_{0} \kappa_{1},
\end{aligned}
$$



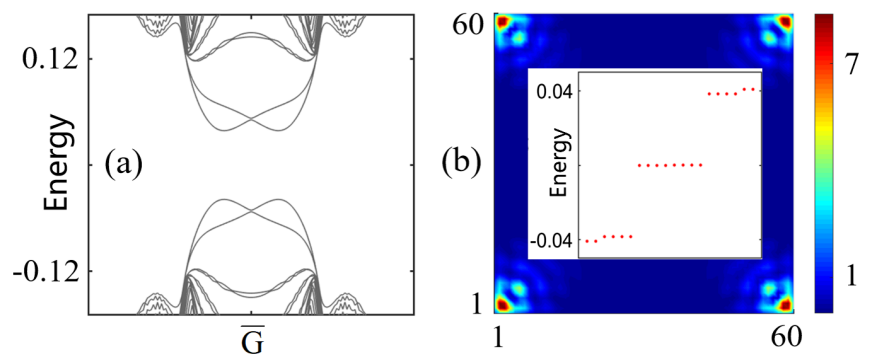

(c)
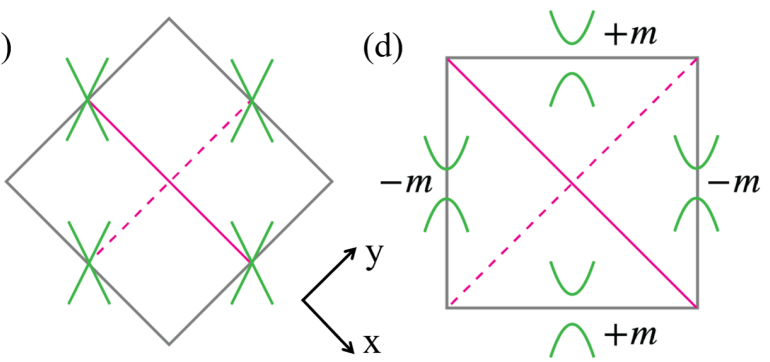

FIG. 4. (a) shows the edge modes on the (11) boundary in the same condition as those in Fig. 3(a). (b) shows the wave-function profiles of the zero-energy modes in the real space under openboundary conditions in both the [11] and [11] directions, with the low-energy spectrum presented in the inset. The color bar in (b) is in the unit of $10^{-3}$. The origin of the corner Majorana modes is sketched in (c) and (d). The magenta (dashed) line labels the mirror symmetry $\left\{M_{y} \mid \mathbf{0}\right\}\left(\left\{M_{x} \mid \mathbf{0}\right\}\right)$. (c) shows the Dirac cones on the $(10) /(01)$ edge protected by $\left\{M_{y} \mid \mathbf{0}\right\} /\left\{M_{x} \mid \mathbf{0}\right\}$. Notice that we use the green lines to label the two Dirac cones on each edge for simplicity. When the edges evolve into the condition in (d), i.e., the (11) and $(1 \overline{1})$ boundaries, on each edge the mirror symmetry no longer preserves and the Dirac cones gain a mass term. The mass terms on different edges are odd with respect to the mirror symmetries $\left\{M_{y} \mid \mathbf{0}\right\}$ and $\left\{M_{x} \mid \mathbf{0}\right\}$ and form the configuration in (d). At the corners, the sign-changed mass terms lead to the zeroenergy modes.

with $m_{(11) /(1 \overline{1})}$ the mass term on the $(11) /(1 \overline{1})$ edge. Moreover, the mirror symmetry $\left\{M_{y} \mid \mathbf{0}\right\}$ demands $m_{(11)}=-m_{(1 \overline{1})}$ (details in Appendix E). Obviously, the theory in Eq. (11) describes a massive Dirac theory, whose mass changes sign at the intersection between the (11) and (11) edges. This mass domain leads to a pair of Majorana modes localized at the corner [84-87], consistent with the results in Fig. 4(b). Based on the analysis, it is clear that, besides the intersection between the (11) and (11) edges, any corner respecting the mirror symmetry $\left\{M_{y} \mid \mathbf{0}\right\}$ would support the Majorana modes. Moreover, under a general condition with $\left|w_{Y}^{ \pm i}\right|=2 \mathcal{Z}$, we can conclude that $\mathcal{Z}$ Majorana Kramers' pairs would arise at the corner. Similar analysis can be applied to the mirror symmetry $\left\{M_{x} \mid \mathbf{0}\right\}$, and we can obtain the mass term configuration in Fig. 4(d). At each corner, there is a symmetryenforced sign change in the mass terms, leading to the corner Majorana modes in Fig. 4(b).

\section{GENERALIZATION TO 3D SUPERCONDUCTORS}

The above analysis in 2D lattices can be generalized to the 3D case. Specifically, we can stack the lattice in Fig. 1(a) along the $z$ direction, in which condition the space group $P 4 / \mathrm{nmm}$ is preserved, and consider the edge modes on the (100) surface and the hinge modes at the intersection between the (110) and (11 0$)$ surfaces. We can calculate the winding numbers protected by the mirror symmetry $\left\{M_{y} \mid \mathbf{0}\right\}, w_{0}^{ \pm i}\left(k_{z}^{0}\right)$ on line $\left(k_{x}, 0, k_{z}^{0}\right)$ and $w_{\pi}^{ \pm i}\left(k_{z}^{0}\right)$ on line $\left(k_{x}, \pi, k_{z}^{0}\right)$ for each fixed $k_{z}^{0}$. Notice that the winding number is well defined on these lines, because the chiral symmetry leaves each $\mathbf{k}$ point unchanged in the Brillouin zone. Correspondingly, $\left|w_{0 / \pi}^{ \pm i}\left(k_{z}^{0}\right)\right|$ pairs of zero-energy modes can be found at $\left(0 / \pi, k_{z}^{0}\right)$ in the (100) surface Brillouin zone labeled by $\left(k_{y}, k_{z}\right)$. Moreover, the change of the winding numbers is closely related to the gapless points in the bulk energy spectrum. For instance, we consider the winding numbers on the $L_{1}$ and $L_{2}$ lines in Fig. 5(a), assuming $w_{\pi}^{ \pm i}\left(k_{z}^{1}\right)$ and $w_{\pi}^{ \pm i}\left(k_{z}^{2}\right)$. If $w_{\pi}^{ \pm i}\left(k_{z}^{1}\right) \neq w_{\pi}^{ \pm i}\left(k_{z}^{2}\right)$, the winding number goes through a topological phase transition in the process $k_{z}^{1}$ evolving into $k_{z}^{2}$, leading to gapless points in the region $k_{z}^{2}<k_{z}<k_{z}^{1}$ in the $k_{y}=\pi$ plane in the bulk spectrum.

On the other hand, similar to the $2 \mathrm{D}$ case, the symmetry $\left\{I \mid \tau_{\mathbf{0}}\right\} \Theta$ requires a Bloch state with eigenvalue $m_{y}$ to be degenerate with a state $-m_{y}$ in the $k_{y}=0$ plane and be degenerate with a state $m_{y}$ in the $k_{y}=\pi$ plane. Taking the rotational symmetry $\left\{C_{2 z} \mid \mathbf{0}\right\}$ into account, one immediately realizes that the condition of the normal bands along each line $\left(k_{x}, 0 / \pi, k_{z}^{0}\right)$ is qualitatively the same as those in Figs. 1(c) and 1(d). Therefore, for the $s$-wave pairing state, the winding number $w_{0 / \pi}^{ \pm i}\left(k_{z}^{0}\right)$ on line $\left(k_{x}, 0 / \pi, k_{z}^{0}\right)$ can be analyzed in a similar way as the 2D case. More specifically, we have $w_{0}^{i}\left(k_{z}^{0}\right)=-w_{0}^{-i}\left(k_{z}^{0}\right)=0$ regardless of pairing orders, while $w_{\pi}^{i}\left(k_{z}^{0}\right)=-w_{\pi}^{-i}\left(k_{z}^{0}\right)=2 \mathcal{Z}$ in the extended $s$-wave state. Moreover, the edge and corner modes for each $k_{z}^{0}$ are also expected to be similar to those in the 2D case.

Based on the above analysis, we can conclude that in 3D superconductors there exist three different phases [88]: a full-gap trivial phase, a full-gap second-order topological superconductor phase sketched in Figs. 5(d) and 5(e), and a gapless second-order topological superconductor phase with Dirac nodes in the bulk energy spectrum sketched in Figs. 5(b) and 5(c), which can be dubbed as the secondorder topological Dirac superconductor phase [58]. In the two topological phases, $2 \mathcal{Z}$ degenerate quasi-1D Dirac cones on the (100) surface and $\mathcal{Z}$ pairs of flat Majorana hinge modes at the corner between the (110) and (110) surfaces arise. Notice that, in Figs. 5(b)-5(e), we assume that there are no accidental nodes in the bulk and the 2D subsystem within the $k_{z}=0$ plane is topologically 


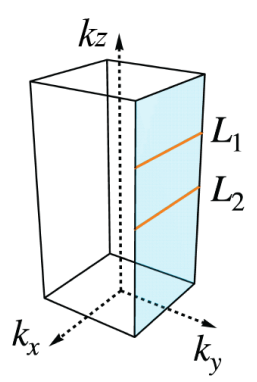

(a)

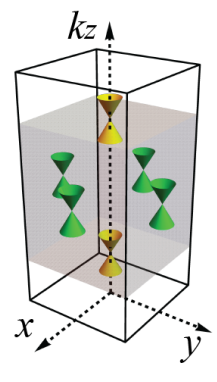

(b)

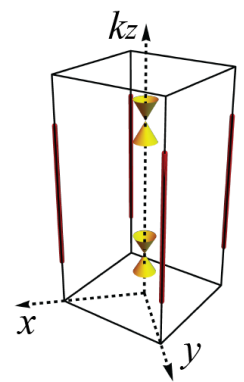

(c)

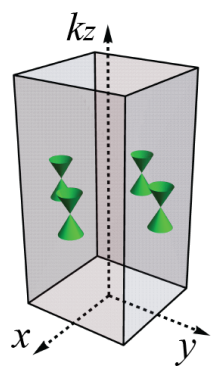

(d)

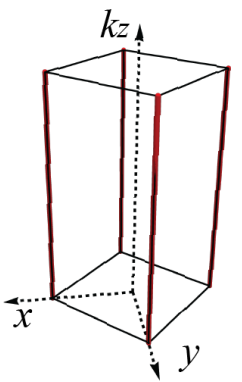

(e)

FIG. 5. (a) shows the first Brillouin zone corresponding to the $3 \mathrm{D}$ lattice respecting the $P 4 / \mathrm{nmm}$ symmetry group. $L_{1}$ and $L_{2}$ (orange) are two lines in the $k_{y}=\pi$ plane (cyan). (b)-(e) illustrate different topological superconductor phases in the system. (b) and (c) sketch the second-order topological Dirac superconductor phase. In this phase, there are Dirac nodes (yellow) in the bulk at $\left(k_{x}^{0}, \pi, k_{z}^{0}\right)$ and its symmetry-related points. On the (100) surface shown in (b), there are $2 \mathcal{Z}$ Dirac cones (green) dispersing flatly along the line $k_{y}=\pi$ for $\left|k_{z}\right|<k_{z}^{0}$ (the gray region); and at the hinges between the (11) and (11) surfaces shown in (c), there are $\mathcal{Z}$ Majorana pairs (red) dispersing flatly along the $k_{z}$ direction for $\left|k_{z}\right|<k_{z}^{0}$. (d) and (e) sketch the full-gap second-order topological superconductor phase. In this condition, $2 \mathcal{Z}$ Dirac cones (green) disperse flatly across the surface Brillouin zone along the $k_{y}=\pi$ line on the (100) surface shown in (d); and $\mathcal{Z}$ Majorana pairs (red) disperse flatly across the 1D Brillouin zone at the hinges between the (11) and (1 $\overline{1})$ surfaces shown in (e). The modes on other edges and hinges can be obtained by symmetry and are also indicated in (b)-(e).

nontrivial. These conclusions are further verified by numerical simulations given in Appendix F.

\section{IMPLICATION TO THE SUPERCONDUCTING STATE IN IRON-BASED SUPERCONDUCTORS}

In iron-based superconductors, in particular, in those iron chalcogenides, such as $\mathrm{KFe}_{2} \mathrm{Se}_{2}[89,90]$, the monolayer $\mathrm{FeSe} / \mathrm{STO}$ [91], and $\mathrm{LiFe}(\mathrm{OH}) \mathrm{FeSe}$ [92], where their Fermi surfaces are featured by two electronic pockets at the Brillouin zone corners as shown in Fig. 6, there is a longlasting debate about the pairing symmetry in their superconducting states [68,93-95]. High-resolution angleresolved photoemission spectroscopy and scanning tunneling spectroscopy (STM) measurements reveal a nodeless superconducting gap [89-92,96] in these materials.

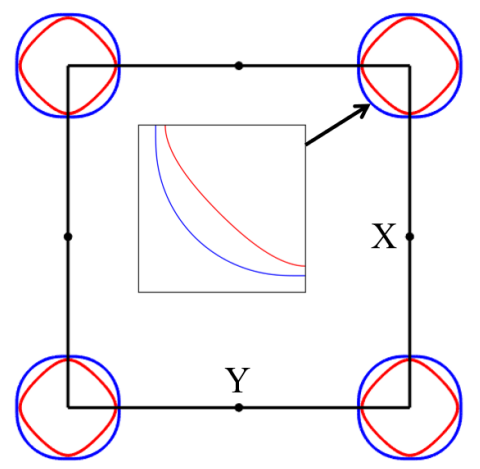

FIG. 6. The typical Fermi surfaces in the monolayer FeSe or $\mathrm{LiFe}(\mathrm{OH}) \mathrm{FeSe}$ in the presence of spin-orbit coupling, with the details shown in the inset. A sign reverse between the superconducting orders on the inner and outer Fermi surfaces, which leads to topological superconductivity as shown in Fig. 3(a), are indicated by the red and blue colors in the figure.
Although the results seem to be consistent with a conventional $s$-wave state [97], it has also been argued that a signchanged $s$-wave state $[67,98,99]$ between the inner and outer pockets can be a candidate as well. In the presence of strong spin-orbit coupling or interband pairing $[67,70,100,101]$, the latter can be nodeless. The experimental results on this issue remain highly controversial with supporting evidence for both cases. Indirect experimental evidence from inelastic neutron scattering [93] and STM quasiparticle interference [102] exists for the signchanged $s$-wave state, while impurity scattering suggests the conventional $s$-wave state $[97,103]$.

The Fermi surfaces in Fig. 6 are qualitatively the same as that in Fig. 3(a). Thus, if the iron chalcogenides are in the sign-changed $s$-wave states, according to the criterion in Eq. (8), we can expect that they are second-order topological superconductors and must exhibit two Dirac cones on the $(10) /(01)$ boundary and one Majorana Kramers'

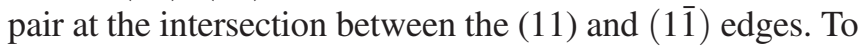
demonstrate this, we carry out our calculation in a fiveorbital model which describes the genuine band structures of iron-based superconductors [104]. The result is given in Appendix G, which confirms that iron chalcogenides, if they host sign-changed $s$-wave states between two electron pockets, are second-order topological superconductors.

This results offers smoking-gun evidence to reveal the pairing nature of iron-based superconductors [105]. The monolayer $\mathrm{FeSe}$ and $\mathrm{LiFe}(\mathrm{OH}) \mathrm{FeSe}$ are the two best systems for such a purpose, as their superconducting gaps can be more than $10 \mathrm{meV}$. If they are in the sign-changed $s$ wave state, a local probe, such as STM, can easily capture the boundary-selective Dirac cones on the $(10) /(11)$ boundaries. A tunneling measurement at the intersection between the (11) and (1 $\overline{1})$ edge can measure the quantized zero-bias conductance peak of $4 e^{2} / h$ resulting from Majorana Kramers' pairs [17]. 


\section{CONCLUSIONS}

In summary, we identify that the centrosymmetric superconductor, which respects a nonsymmorphic symmetry group, can host topological superconductivity even though its superconducting order belongs to an $s$-wave state [106]. The anomalous band degeneracies stemming from nonsymmorphic symmetries are vital in realizing topological superconductivity. The study is specified with the space group $P 4 / \mathrm{nmm}$, with a Fermi surface criterion developed for the topological superconductivity. Based on the analysis, we reveal that the iron-chalcogenide superconductors, if they are in the sign-changed $s$-wave state $[67,68]$, are second-order topological superconductors, which have two Dirac cones on the $(10) /(01)$ boundary and one Majorana Kramers' pair at the intersection between the (11) and (11) edges. Therefore, by measuring the topological property, in a similar spirit as previous studies [17,69], we can reveal essential information on the pairing symmetry of the iron-chalcogenide superconductors. Our study uncovers a new direction in the pursuit of the topological superconductors and establishes a measurable quantity to settle one long-lasting debate on the pairing nature of iron-based superconductors.

\section{ACKNOWLEDGMENTS}

The authors are grateful to Xianxin $\mathrm{Wu}$ and Zhongyi Zhang for fruitful discussions. This work is supported by the Ministry of Science and Technology of China 973 program (Grants No. 2017YFA0303100 and No. 2016YFA0302400), National Science Foundation of China (Grants No. NSFC-11888101 and No. NSFC11920101005), and the Strategic Priority Research Program of Chinese Academy of Sciences (Grants No. XDB33000000 and No. XDB28000000).

\section{APPENDIX A: QUOTIENT GROUP $G / T$ OF THE SPACE GROUP P4/nmm}

As mentioned in the main text, there are totally 16 symmetry operations in the quotient group $G / T$ of the space group $P 4 / \mathrm{nmm}$. We list these operations defined in the lattice in Fig. 1(a) in the main text in the following:

$$
\begin{aligned}
& \{E \mid \mathbf{0}\}, \quad\left\{C_{2 z} \mid \mathbf{0}\right\}, \quad\left\{C_{2 x y} \mid \mathbf{0}\right\}, \quad\left\{C_{2 \bar{x} y} \mid \mathbf{0}\right\}, \quad\left\{M_{x} \mid \mathbf{0}\right\}, \quad\left\{M_{y} \mid \mathbf{0}\right\}, \quad\left\{S_{4 z} \mid \mathbf{0}\right\}, \quad\left\{S_{4 z}^{-1} \mid \mathbf{0}\right\}, \\
& \left\{I \mid \tau_{\mathbf{0}}\right\}, \quad\left\{M_{z} \mid \tau_{\mathbf{0}}\right\}, \quad\left\{M_{x y} \mid \tau_{\mathbf{0}}\right\}, \quad\left\{M_{\bar{x} y} \mid \tau_{\mathbf{0}}\right\}, \quad\left\{C_{2 x} \mid \tau_{\mathbf{0}}\right\}, \quad\left\{C_{2 y} \mid \tau_{\mathbf{0}}\right\}, \quad\left\{C_{4 z}^{-1} \mid \tau_{\mathbf{0}}\right\}, \quad\left\{C_{4 z} \mid \tau_{\mathbf{0}}\right\},
\end{aligned}
$$

where $\tau_{\mathbf{0}}=\mathbf{a}_{1} / 2+\mathbf{a}_{2} / 2$ and all the symmetry operations are defined at the lattice site. The point group part of the above operations, i.e., $\alpha$ in the Seitz operator $\{\alpha \mid \boldsymbol{\tau}\}$, acts on the coordinates in Fig. 1(a) in the main text as follows:

$$
\begin{aligned}
& E:(x, y, z) \mapsto(x, y, z), \quad C_{2 z}:(x, y, z) \mapsto(-x,-y, z), \quad C_{2 x y}:(x, y, z) \mapsto(y, x,-z), \quad C_{2 \bar{x} y}:(x, y, z) \mapsto(-y,-x,-z), \\
& M_{x}:(x, y, z) \mapsto(-x, y, z), \quad M_{y}:(x, y, z) \mapsto(x,-y, z), \quad S_{4 z}:(x, y, z) \mapsto(y,-x,-z), \quad S_{4 z}^{-1}:(x, y, z) \mapsto(-y, x,-z), \\
& I:(x, y, z) \mapsto(-x,-y,-z), \quad M_{z}:(x, y, z) \mapsto(x, y,-z), \quad M_{x y}:(x, y, z) \mapsto(-y,-x, z), \quad M_{\bar{x} y}:(x, y, z) \mapsto(y, x, z), \\
& C_{2 x}:(x, y, z) \mapsto(x,-y,-z), \quad C_{2 y}:(x, y, z) \mapsto(-x, y,-z), \quad C_{4 z}^{-1}:(x, y, z) \mapsto(-y, x, z), \quad C_{4 z}:(x, y, z) \mapsto(y,-x, z) .
\end{aligned}
$$

\section{APPENDIX B: MIRROR SYMMETRY IN SPACE GROUP P4/nmm}

In the main text, we show that the band degeneracies along the $\Sigma_{Y}$ and $\Sigma_{G}$ lines are different, based on the analysis of the group structure of $P 4 / \mathrm{nmm}$. Here, we provide a detailed calculation by decoupling the normal-state lattice Hamiltonian in the main text according to the mirror symmetry $\left\{M_{y} \mid \mathbf{0}\right\}$.

We first rotate the spin to the $y$ direction. Namely, we do a unitary transformation $\mathcal{H}_{0}^{\prime}=e^{-i \pi / 4 s_{1}} \mathcal{H}_{0} e^{i \pi / 4 s_{1}}$. In $\mathcal{H}_{0}^{\prime}$, the spins $\uparrow \downarrow$ are along the $y$ direction and $p_{x / y}$ are eigenstates of $\left\{M_{y} \mid \mathbf{0}\right\}$. Therefore, in general, $\left(c_{A x \uparrow}, c_{A y \downarrow}, c_{B x \uparrow}, c_{B y \downarrow}\right)\left(c_{A x \uparrow}\right.$ is the annihilation operator for the spin- $\uparrow p_{x}$-orbital electron in the $A$ sublattice) would be in a mirror invariant subspace, while their time reversal partners are in the other mirror subspace. On the line $\Sigma_{G}$ for group $P 4 / \mathrm{nmm}$, we can write down $\mathcal{H}_{0}^{\prime}$ as 


$\left(\begin{array}{cccccccc}2 t \cos k_{x}-2 \lambda_{R} \sin k_{x} & 0 & 0 & \frac{\lambda}{2} & 4 t_{1} \cos \frac{k_{x}}{2} & 0 & 0 & 0 \\ 0 & 2 t & -\frac{\lambda}{2} & 0 & 0 & 4 t_{1} \cos \frac{k_{x}}{2} & 0 & 0 \\ 0 & -\frac{\lambda}{2} & 2 t \cos k_{x}+2 \lambda_{R} \sin k_{x} & 0 & 0 & 0 & 4 t_{1} \cos \frac{k_{x}}{2} & 0 \\ \frac{\lambda}{2} & 0 & 0 & 2 t & 0 & 0 & 0 & 4 t_{1} \cos \frac{k_{x}}{2} \\ 4 t_{1} \cos \frac{k_{x}}{2} & 0 & 0 & 0 & 2 t \cos k_{x}+2 \lambda_{R} \sin k_{x} & 0 & 0 & \frac{\lambda}{2} \\ 0 & 4 t_{1} \cos \frac{k_{x}}{2} & 0 & 0 & 0 & 2 t & -\frac{\lambda}{2} & 0 \\ 0 & 0 & 4 t_{1} \cos \frac{k_{x}}{2} & 0 & 0 & -\frac{\lambda}{2} & 2 t \cos k_{x}-2 \lambda_{R} \sin k_{x} & 0 \\ 0 & 0 & 0 & 4 t_{1} \cos \frac{k_{x}}{2} & \frac{\lambda}{2} & 0 & 0 & 2 t\end{array}\right)$,

with the basis $\left(c_{A x \uparrow}, c_{A y \uparrow}, c_{A x \downarrow}, c_{A y \downarrow}, c_{B x \uparrow}, c_{B y \uparrow}, c_{B x \downarrow}, c_{B y \downarrow}\right)$. As analyzed, $\left(c_{A x \uparrow}, c_{A y \downarrow}, c_{B x \uparrow}, c_{B y \downarrow}\right)$ are in a mirror invariant subspace. The situation is very different on line $\Sigma_{Y}$. Under the same basis with the Hamiltonian in Eq. (B1), $\mathcal{H}_{0}^{\prime}$ on $\Sigma_{Y}$ takes the form

$$
\left(\begin{array}{cccccccc}
2 t \cos k_{x}-2 \lambda_{R} \sin k_{x} & 0 & 0 & \frac{\lambda}{2} & 0 & -4 t_{2} \sin \frac{k_{x}}{2} & 0 & 0 \\
0 & 2 t & -\frac{\lambda}{2} & 0 & -4 t_{2} \sin \frac{k_{x}}{2} & 0 & 0 & 0 \\
0 & -\frac{\lambda}{2} & 2 t \cos k_{x}+2 \lambda_{R} \sin k_{x} & 0 & 0 & 0 & 0 & -4 t_{2} \sin \frac{k_{x}}{2} \\
\frac{\lambda}{2} & 0 & 0 & 2 t & 0 & 0 & -4 t_{2} \sin \frac{k_{x}}{2} & 0 \\
0 & -4 t_{2} \sin \frac{k_{x}}{2} & 0 & 0 & 2 t \cos k_{x}+2 \lambda_{R} \sin k_{x} & 0 & 0 & \frac{\lambda}{2} \\
-4 t_{2} \sin \frac{k_{x}}{2} & 0 & 0 & 0 & 0 & 2 t & -\frac{\lambda}{2} & 0 \\
0 & 0 & 0 & -4 t_{2} \sin \frac{k_{x}}{2} & 0 & -\frac{\lambda}{2} & 2 t \cos k_{x}-2 \lambda_{R} \sin k_{x} & 0 \\
0 & 0 & -4 t_{2} \sin \frac{k_{x}}{2} & 0 & \frac{\lambda}{2} & 0 & 0 & 2 t
\end{array}\right) .
$$

In this case, $\left(c_{A x \uparrow}, c_{A y \downarrow}, c_{B y \uparrow}, c_{B x \downarrow}\right)$ are in a mirror invariant subspace. We apply the symmetry $\left\{I \mid \tau_{\mathbf{0}}\right\} \Theta$ on the mirror invariant subspace

$$
\begin{aligned}
& \Sigma_{G}:\left(c_{A x \uparrow}, c_{A y \downarrow}, c_{B x \uparrow}, c_{B y \downarrow}\right) \stackrel{\left\{I \mid \tau_{0}\right\}}{\longrightarrow}\left(c_{B x \uparrow}, c_{B y \downarrow}, c_{A x \uparrow}, c_{A y \downarrow}\right) \stackrel{\Theta}{\longrightarrow}\left(c_{B x \downarrow}, c_{B y \uparrow}, c_{A x \downarrow}, c_{A y \uparrow}\right), \\
& \Sigma_{Y}:\left(c_{A x \uparrow}, c_{A y \downarrow}, c_{B y \uparrow}, c_{B x \downarrow}\right) \stackrel{\left\{I \mid \tau_{0}\right\}}{\longrightarrow}\left(c_{B x \uparrow}, c_{B y \downarrow}, c_{A y \uparrow}, c_{A x \downarrow}\right) \stackrel{\Theta}{\longrightarrow}\left(c_{B x \downarrow}, c_{B y \uparrow}, c_{A y \downarrow}, c_{A x \uparrow}\right) .
\end{aligned}
$$

In the above equation, we take advantage of the fact that the inversion symmetry interchanges the two sublattices but preserves spin, while the time reversal symmetry merely flips the local spin. According to Eq. (B3), $\left\{I \mid \tau_{\mathbf{0}}\right\} \Theta$ maps the $\left\{M_{y} \mid \mathbf{0}\right\}$ flips of the two $\left\{M_{y} \mid \mathbf{0}\right\}$ subspaces on $\Sigma_{G}$ while it preserves in each of the subspaces on $\Sigma_{Y}$, which is consistent with the general symmetry analysis in the main text.

The above difference can be straightforwardly understood by considering the Fourier transform of the real-space basis

$$
\left|\phi_{A}(\mathbf{k})\right\rangle=\sum_{j} e^{i \mathbf{k} \cdot \mathbf{R}_{A}^{j}}\left|\phi_{A}\left(\mathbf{R}_{A}^{j}\right)\right\rangle, \quad\left|\phi_{B}(\mathbf{k})\right\rangle=\sum_{j} e^{i \mathbf{k} \cdot \mathbf{R}_{B}^{j}}\left|\phi_{B}\left(\mathbf{R}_{B}^{j}\right)\right\rangle
$$

where $\mathbf{R}_{A / B}^{j}$ is the position of the $A / B$ site in the $j$ th unit cell, with $\mathbf{R}_{A}^{j}-\mathbf{R}_{B}^{j}=\tau_{\mathbf{0}}$. Applying the mirror symmetry $\left(k_{y} \rightarrow-k_{y}\right)$, we have

$$
k_{y}=\pi:\left\{M_{y} \mid \mathbf{0}\right\}\left|\phi_{A}(\mathbf{k})\right\rangle=\sum_{j} e^{i \mathbf{k} \cdot \mathbf{R}_{A}^{j}} m_{A}\left|\phi_{A}\left(\mathbf{R}_{A}^{j}\right)\right\rangle, \quad\left\{M_{y} \mid \mathbf{0}\right\}\left|\phi_{B}(\mathbf{k})\right\rangle=-\sum_{j} e^{i \mathbf{k} \cdot \mathbf{R}_{B}^{j}} m_{B}\left|\phi_{B}\left(\mathbf{R}_{B}^{j}\right)\right\rangle,
$$




$$
k_{y}=0:\left\{M_{y} \mid \mathbf{0}\right\}\left|\phi_{A}(\mathbf{k})\right\rangle=\sum_{j} e^{i \mathbf{k} \cdot \mathbf{R}_{A}^{j}} m_{A}\left|\phi_{A}\left(\mathbf{R}_{A}^{j}\right)\right\rangle, \quad\left\{M_{y} \mid \mathbf{0}\right\}\left|\phi_{B}(\mathbf{k})\right\rangle=\sum_{j} e^{i \mathbf{k} \cdot \mathbf{R}_{B}^{j}} m_{B}\left|\phi_{B}\left(\mathbf{R}_{B}^{j}\right)\right\rangle,
$$

with $m_{A / B}$ the mirror eigenvalues. In the equation, we consider the fact that $R_{A / B, y}^{j} \cdot k_{y}=j \pi$ at the Brillouin zone boundary (definition of $k_{y}$ ). The results in Eq. (B5) explain the difference between the mirror invariant subspaces in Eqs. (B1) and (B2).

\section{APPENDIX C: SYMMETRY CONSTRAINTS ON THE WINDING NUMBER}

In this part, we show how the symmetries constrain the winding number. As mentioned in the main text, for a 1D system $\mathcal{H}$ with chiral symmetry $\mathcal{C}$, its topological property can be characterized by the winding number $w$. If the system has additional symmetry $g$ satisfying $g \mathcal{H}(k) g^{-1}=\mathcal{H}(\mathbf{g} k)$, the symmetry has a constraint on $w$ as follows [107]:

$$
\begin{aligned}
w & =\int_{-\pi}^{\pi} \frac{d k}{2 \pi} \operatorname{tr}\left[\mathcal{C H}^{-1}(k) \partial_{k} \mathcal{H}(k)\right] \\
& =\int_{-\pi}^{\pi} \frac{d k}{2 \pi} \operatorname{tr}\left\{\mathcal{C}\left[g \mathcal{H}\left(\mathbf{g}^{-1} k\right) g^{-1}\right]^{-1} \partial_{k}\left[g \mathcal{H}\left(\mathbf{g}^{-1} k\right) g^{-1}\right]\right\} \\
& =\int_{-\pi}^{\pi} \frac{d k}{2 \pi} \operatorname{tr}\left[g^{-1} \mathcal{C} g \mathcal{H}^{-1}\left(\mathbf{g}^{-1} k\right) \partial_{k} \mathcal{H}\left(\mathbf{g}^{-1} k\right)\right] \\
& =\int_{-g^{-1} \pi}^{g^{-1} \pi} \frac{d(\mathbf{g} k)}{2 \pi} \operatorname{tr}\left[g^{-1} \mathcal{C} g \mathcal{H}^{-1}(k) \partial_{\mathbf{g} k} \mathcal{H}(k)\right] \\
& =\int_{-\pi}^{\pi} \frac{d k}{2 \pi} \operatorname{tr}\left[g^{-1} \mathcal{C} g \mathcal{H}^{-1}(k) \partial_{k} \mathcal{H}(k)\right] \cdot \operatorname{det}(\mathbf{g}) \\
& =\int_{-\pi}^{\pi} \frac{d k}{2 \pi} \operatorname{tr}\left[\mathcal{C H}^{-1}(k) \partial_{k} \mathcal{H}(k)\right] \cdot \operatorname{det}(\mathbf{g}),
\end{aligned}
$$

where we use the property $[\mathcal{C}, g]=0$ for the $s$-wave superconductivity.

Now we consider the winding number in each of the $\left\{M_{y} \mid \mathbf{0}\right\}$ subspaces on the $\Sigma_{Y / G}$ line. As mentioned in the main text, the bands are always twofold degenerate due to the presence of the symmetry $\left\{I \mid \tau_{0}\right\} \Theta$. Moreover, the symmetries on the $\Sigma_{Y / G}$ line require

$$
\begin{gathered}
\left(\left\{I \mid \tau_{\mathbf{0}}\right\} \Theta\right)\left\{M_{z} \mid \tau_{\mathbf{0}}\right\}|\varphi(\mathbf{k})\rangle=e^{-2 i \mathbf{k} \cdot \tau_{\mathbf{0}}}\left\{M_{z} \mid \tau_{\mathbf{0}}\right\}\left(\left\{I \mid \tau_{\mathbf{0}}\right\} \Theta\right)|\varphi(\mathbf{k})\rangle, \\
\left(\left\{I \mid \tau_{\mathbf{0}}\right\} \Theta\right)\left\{M_{y} \mid \mathbf{0}\right\}|\varphi(\mathbf{k})\rangle=e^{i k_{y}}\left\{M_{y} \mid \mathbf{0}\right\}\left(\left\{I \mid \tau_{\mathbf{0}}\right\} \Theta\right)|\varphi(\mathbf{k})\rangle, \\
\left\{M_{z} \mid \tau_{\mathbf{0}}\right\}\left\{M_{y} \mid \mathbf{0}\right\}|\varphi(\mathbf{k})\rangle=-e^{i k_{y}}\left\{M_{y} \mid \mathbf{0}\right\}\left\{M_{z} \mid \tau_{\mathbf{0}}\right\}|\varphi(\mathbf{k})\rangle,
\end{gathered}
$$

namely,

$$
\begin{array}{ll}
k_{y}=\pi:\left[\left\{I \mid \tau_{\mathbf{0}}\right\} \Theta,\left\{M_{y} \mid \mathbf{0}\right\}\right]_{+}=0, & {\left[\left\{M_{z} \mid \tau_{\mathbf{0}}\right\},\left\{M_{y} \mid \mathbf{0}\right\}\right]_{-}=0,} \\
k_{y}=0:\left[\left\{I \mid \tau_{\mathbf{0}}\right\} \Theta,\left\{M_{y} \mid \mathbf{0}\right\}\right]_{-}=0, & {\left[\left\{M_{z} \mid \tau_{\mathbf{0}}\right\},\left\{M_{y} \mid \mathbf{0}\right\}\right]_{+}=0 .}
\end{array}
$$

Based on the above constraints, we can obtain the following conclusions.

(i) On $\Sigma_{Y}$, the two degenerate states are in the same mirror subspace which can be further decoupled by $\left\{M_{z} \mid \tau_{\mathbf{0}}\right\}$. Since the two states are related by the symmetry $\left\{I \mid \tau_{\mathbf{0}}\right\} \Theta$, we have $w_{Y}^{m_{y}, m_{z}}=w_{Y}^{m_{y},-m_{z}}$, with $m_{y}= \pm i$ and $m_{z}= \pm i e^{i \mathbf{k} \cdot \tau_{0}}$ the eigenvalues of $\left\{M_{y} \mid \mathbf{0}\right\}$ and $\left\{M_{z} \mid \tau_{\mathbf{0}}\right\}$, respectively. Considering the constraint of the time reversal symmetry, we have $w_{Y}^{+i}=-w_{Y}^{-i}=2 \mathcal{Z}$.

(ii) On $\Sigma_{G}$, we take the inversion symmetry $\left\{I \mid \tau_{\mathbf{0}}\right\}$ into consideration, which satisfies $\left\{M_{y} \mid \mathbf{0}\right\}\left\{I \mid \tau_{\mathbf{0}}\right\}|\varphi(\mathbf{k})\rangle=$ $e^{i k_{y}}\left\{I \mid \tau_{\mathbf{0}}\right\}\left\{M_{y} \mid \mathbf{0}\right\}|\varphi(\mathbf{k})\rangle=\left\{I \mid \tau_{\mathbf{0}}\right\}\left\{M_{y} \mid \mathbf{0}\right\}|\varphi(\mathbf{k})\rangle$. Obviously, $\left\{I \mid \tau_{\mathbf{0}}\right\}$ maps a state at $\mathbf{k}$ to a state at $-\mathbf{k}$ in the same mirror subspace. According to Eq. (C1), we have $w_{G}^{+i}=-w_{G}^{-i}=0$. 


\section{APPENDIX D: TOPOLOGICAL TRIVIALNESS IN CENTROSYMMETRIC SYMMORPHIC SUPERCONDUCTORS WITH $s$-WAVE $\left(A_{1 g}\right)$ PAIRING}

In this part, we show that in centrosymmetric superconductors respecting symmorphic groups, if the pairing order is in the $A_{1 g}$ channel, the superconductivity is always topologically trivial with respect to (i) the topological indices in the Altland-Zirnbauer (AZ) classification and (ii) the mirror-protected topological indices (the mirror Chern number and mirror winding number).

\section{Without crystalline symmetry}

We begin with the topological indices in the $A Z$ classification. In the $1 \mathrm{D}$ and $3 \mathrm{D}$ cases, the class-DIII superconductors are featured by the $\mathcal{Z}$ indices, which are the 1D and 3D winding numbers defined according to the chiral symmetry $\mathcal{C}$. We label the inversion symmetry with $\mathcal{I}$. As mentioned, for $A_{1 g}$ superconductivity $[\mathcal{C}, \mathcal{I}]=0$. Moreover, since $\mathcal{I} \mathbf{k}=-\mathbf{k}$, namely, $\operatorname{det}(\mathcal{I})=-1$. Hence, in the $1 \mathrm{D}$ case, $w_{1 \mathrm{D}}=0$ according to Eq. (C1). The $3 \mathrm{D}$ case can be analyzed similarly. In the presence of inversion symmetry, the $3 \mathrm{D}$ winding number is confined as

$$
\begin{aligned}
w_{3 \mathrm{D}}= & \int \frac{d^{3} k}{48 \pi^{2}} \epsilon^{\alpha \beta \gamma} \operatorname{tr}\left[\mathcal{C H}^{-1}(k) \partial_{k_{\alpha}} \mathcal{H}(k) \mathcal{H}^{-1}(k) \partial_{k_{\beta}} \mathcal{H}(k) \mathcal{H}^{-1}(k) \partial_{k_{\gamma}} \mathcal{H}(k)\right] \\
= & \int \frac{d^{3} k}{48 \pi^{2}} \epsilon^{\alpha \beta \gamma} \operatorname{tr}\left\{\mathcal{C}\left[\mathcal{I} \mathcal{H}(-\mathbf{k}) \mathcal{I}^{-1}\right]^{-1} \partial_{k_{\alpha}}\left[\mathcal{I} \mathcal{H}(-\mathbf{k}) \mathcal{I}^{-1}\right]\left[\mathcal{I} \mathcal{H}(-\mathbf{k}) \mathcal{I}^{-1}\right]^{-1}\right. \\
& \left.\times \partial_{k_{\beta}}\left[\mathcal{I} \mathcal{H}(-\mathbf{k}) \mathcal{I}^{-1}\right]\left[\mathcal{I} \mathcal{H}(-\mathbf{k}) \mathcal{I}^{-1}\right]^{-1} \partial_{k_{\gamma}}\left[\mathcal{I} \mathcal{H}(-\mathbf{k}) \mathcal{I}^{-1}\right]\right\} \\
= & \int \frac{d^{3} k}{48 \pi^{2}} \epsilon^{\alpha \beta \gamma} \operatorname{tr}\left[\mathcal{C H}^{-1}(k) \partial_{k_{\alpha}} \mathcal{H}(k) \mathcal{H}^{-1}(k) \partial_{k_{\beta}} \mathcal{H}(k) \mathcal{H}^{-1}(k) \partial_{k_{\gamma}} \mathcal{H}(k)\right] \cdot \operatorname{det}(\mathcal{I}) \\
= & -w_{3 \mathrm{D}}=0,
\end{aligned}
$$

where $[\mathcal{C}, \mathcal{I}]=0$ and $\mathcal{I} \mathbf{k}=-\mathbf{k}$ are taken into consideration. Therefore, in $1 \mathrm{D}$ and $3 \mathrm{D}$, inversion symmetry demands $A_{1 g}$ superconductivity to be topologically trivial.

For the $2 \mathrm{D}$ case, the superconductor is featured by a $\mathcal{Z}_{2}$ index similar to that in a time reversal symmetry-protected topological insulator. In general, it is hard to calculate the $\mathcal{Z}_{2}$ index directly. However, due to the inversion symmetry, we can calculate the $\mathcal{Z}_{2}$ index based on the parity criterion $[108,109]$

$$
\mathcal{Z}_{2}:(-1)^{\nu}=\prod_{i} \prod_{m=1}^{N} \xi_{2 m}\left(\Gamma_{i}\right),
$$

where $\Gamma_{i}(i=1,2,3,4)$ are the four time reversal invariant points in the 2D Brillouin zone and $\xi_{2 m}$ is the parity of the $2 m$ th negative-energy state (time reversal symmetry requires that all of the states appear in the form of Kramers' pairs, and each Kramers' pair shares the same parity). We take the chiral symmetry $\mathcal{C}$ into consideration, which is unitary and satisfies $\mathcal{C H}(\mathbf{k}) \mathcal{C}^{-1}=-\mathcal{H}(\mathbf{k})$. We can realize that $\mathcal{C}$ maps a state $\left[E(\mathbf{k}), \xi_{0}\right]$ [with $E(\mathbf{k})$ the eigenenergy and $\xi_{0}$ the parity) to a state $\left[-E(\mathbf{k}), \xi_{0}\right]$, considering $[\mathcal{C}, \mathcal{I}]=0$. Based on this relation, for $A_{1 g}$ superconductivity, the $\mathcal{Z}_{2}$ index in Eq. (D2) can take only one value. Namely, it is always topologically trivial.

\section{In the presence of mirror symmetry}

We turn to the situation where there is mirror symmetry in a centrosymmetric class-DIII superconductor and consider the mirror-protected topological indices, mainly the mirror Chern number and mirror winding number.

We first consider the condition where there are mirror invariant lines in the Brillouin zone. Specifically, two cases are included: (i) In 1D, the system is parallel to the mirror plane; (ii) in 2D, the mirror plane is normal to the system (for simplicity, we consider a square lattice with mirror symmetry $\mathcal{M}_{y}$ ). In these two cases, we study the mirrorprotected winding numbers, and the analysis is similar to the case of the $\Sigma_{G}$ line in the main text. The winding number in each of the mirror invariant subspaces is always trivial, namely, $w_{1 \mathrm{D}}^{+i}=w_{1 \mathrm{D}}^{-i}=0$ and $w_{2 \mathrm{D}}^{+i}\left(k_{y}=0\right)=$ $w_{2 \mathrm{D}}^{-i}\left(k_{y}=0\right)=w_{2 \mathrm{D}}^{+i}\left(k_{y}=\pi\right)=w_{2 \mathrm{D}}^{-i}\left(k_{y}=\pi\right)=0$.

Then, we consider the condition where there are mirror invariant planes in 3D superconductors (for simplicity, we consider the mirror symmetry $\mathcal{M}_{z}$ in a tetragonal lattice). Within the mirror invariant planes, the mirror Chern numbers are well defined. As pointed out in Ref. [50], the Chern numbers are always zeros in each of the mirror subspaces, $C_{+i}=C_{-i}=0$, because of the chiral symmetry. Moreover, within the mirror invariant planes, the mirror-protected winding number can be considered along the time reversal invariant lines in $k_{z}=0 / \pi$. For instance, the mirror-protected winding number is well defined on lines $\left(k_{x}, k_{y}^{0}, k_{z}^{0}\right)$ and $\left(k_{x}^{0}, k_{y}, k_{z}^{0}\right)$, with $k_{x / y}^{0}=0, \pi$ and $-\pi \leq k_{x / y} \leq \pi$. Because of the inversion symmetry, these mirror-protected winding numbers are always zero, which is similar to the case of the $\Sigma_{G}$ line in the main text. The above analysis is also true for $2 \mathrm{D}$ 
superconductors where the mirror plane is parallel to the system.

\section{APPENDIX E: THEORY FOR THE SYMMETRY- PROTECTED CORNER MODES}

In this part, we (i) further verify the $\mathcal{Z}$ classification of the corner Majorana modes and (ii) derive an effective edge theory to explain that the appearance of corner Majorana modes is guaranteed by mirror symmetry; namely, the topological superconductivity in the main text is an intrinsic second-order one.

\section{1. $\mathcal{Z}$ classification of the corner modes}

We show that the symmetry-protected corner Majorana modes indeed have a $\mathcal{Z}$ classification. This can be verified by considering the hybridization between the corner Majorana modes. We begin with the symmetry constraints, including the mirror symmetry $\mathcal{M}_{y}=\left\{M_{y} \mid \mathbf{0}\right\}$ and the chiral symmetry $\mathcal{C}$, on every single corner mode. As mentioned for the $A_{1 g}$ pairing state, the mirror symmetry $\mathcal{M}_{y}$ commutes with the chiral symmetry $\mathcal{C}$. Therefore, the wave function of the corner mode $|\varphi\rangle$ can be the eigenfunction of both $\mathcal{M}_{y}$ and $\mathcal{C}$. Assuming $\mathcal{M}_{y}|\varphi\rangle=m|\varphi\rangle$ and $\mathcal{C}|\varphi\rangle=c|\varphi\rangle$ with $m= \pm i$ and $c= \pm 1$ the eigenvalues of $\mathcal{M}_{y}$ and $\mathcal{C}$, respectively, we can use the eigenvalues of $\mathcal{M}_{y}$ and $\mathcal{C}$ to label each corner mode $|\varphi\rangle=|m, c\rangle$. For instance, $|+i,+1\rangle$ stands for a corner mode with mirror eigenvalue $+i$ and chiral eigenvalue +1 .

Then, we consider the hybridization between two corner modes $\left|m_{1}, c_{1}\right\rangle$ and $\left|m_{2}, c_{2}\right\rangle$. Obviously, only the corner modes in the same mirror subspace can hybridize, and we consider the $m=-i$ subspace for simplicity here (the condition in the $m=+i$ mirror subspace can be obtained by considering the effect of time reversal symmetry). The Hamiltonian for the system composed of two corner modes $\left|-i, c_{1}\right\rangle$ and $\left|-i, c_{2}\right\rangle$ takes the general form

$$
\mathcal{H}_{\text {hyb }}=a\left|-i, c_{1}\right\rangle\left\langle-i, c_{1}|+b|-i, c_{2}\right\rangle\left\langle-i, c_{2}|+d|-i, c_{1}\right\rangle\left\langle-i, c_{2}\left|+d^{*}\right|-i, c_{2}\right\rangle\left\langle-i, c_{1}\right|,
$$

with $a$ and $b$ real numbers and $d$ complex. Under the chiral symmetry, $\mathcal{H}_{\text {hyb }}$ transforms as

$$
\mathcal{C H}_{\text {hyb }} \mathcal{C}^{-1}=a c_{1}^{2}\left|-i, c_{1}\right\rangle\left\langle-i, c_{1}\left|+b c_{2}^{2}\right|-i, c_{2}\right\rangle\left\langle-i, c_{2}\left|+d c_{1} c_{2}\right|-i, c_{1}\right\rangle\left\langle-i, c_{2}\left|+d^{*} c_{2} c_{1}\right|-i, c_{2}\right\rangle\left\langle-i, c_{1}\right| .
$$

On the other hand, chiral symmetry requires $\mathcal{C H}_{\text {hyb }} \mathcal{C}^{-1}=$ $-\mathcal{H}_{\text {hyb }}$. Straightforwardly, we come to the following conclusions: (i) $a=b=0$; (ii) $d \neq 0$ when and only when $c_{1}=-c_{2}$. Namely, the two corner modes can hybridize and be gapped out when they carry opposite chirality (the eigenvalue of the chiral symmetry), while two corner modes with the same chirality can never hybridize.

The above analysis can be directly generalized to the condition where there are multiple corner modes in the $m=$ $-i$ mirror subspace. If the multiple corner modes carry the same chirality, they cannot hybridize and are stable against the symmetry-preserving perturbations. Considering the requirement of time reversal symmetry, we can conclude

$$
\begin{aligned}
\Theta \mathcal{H}_{\mathrm{eff}, I}\left(k_{y}\right) \Theta^{-1} & =\mathcal{H}_{\mathrm{eff}, I}\left(-k_{y}\right), \\
\mathcal{C} \mathcal{H}_{\mathrm{eff}, I}\left(k_{y}\right) \mathcal{C}^{-1} & =-\mathcal{H}_{\mathrm{eff}, I}\left(k_{y}\right),
\end{aligned}
$$

Moreover, we choose a gauge where the symmetries satisfy

$$
[\Theta, \mathcal{M}]_{-}=[\mathcal{P}, \mathcal{M}]_{-}=\left[\mathcal{C}, \mathcal{M}_{y}\right]_{-}=0 .
$$

We start with the time reversal symmetry and the particlehole symmetry. Without loss of generality, the matrix form of these two symmetries can be chosen as $\Theta=i s_{2} \kappa_{0} K$ and $\mathcal{P}=i s_{2} \kappa_{2} K$ with $K$ the complex conjugate operation. that there can be $\mathcal{Z}$ pairs of corner Majorana modes; namely, the classification in our theory is $\mathcal{Z}$.

\section{Edge theory for the corner modes}

On the (10) edge, the mirror symmetry $\mathcal{M}_{y}$ is preserved. In addition to $\mathcal{M}_{y}$, the particle-hole symmetry $\mathcal{P}$ and the time reversal symmetry $\Theta$ are also preserved. The chiral symmetry $\mathcal{C}=\mathcal{P} \Theta$ also exists on the edge. An effective theory describing the Dirac cones on the edge can be derived based on the constraints of the above four symmetries. These symmetries constrain the edge theory on the (10) edge as

$$
\begin{aligned}
& \mathcal{P} \mathcal{H}_{\text {eff }, I}\left(k_{y}\right) \mathcal{P}^{-1}=-\mathcal{H}_{\text {eff }, I}\left(-k_{y}\right), \\
& \mathcal{M}_{y} \mathcal{H}_{\text {eff }, I}\left(k_{y}\right) \mathcal{M}_{y}^{-1}=\mathcal{H}_{\text {eff }, I}\left(-k_{y}\right) .
\end{aligned}
$$

Here, $s_{i}$ are the Pauli matrices labeling the spin degree of freedom and $\kappa_{i}$ the Pauli matrices labeling the remaining degree for the two Dirac cones on the edge. Correspondingly, the chiral symmetry takes the form $\mathcal{C}=s_{0} \kappa_{2}$. The constraints of time reversal symmetry and particle-hole symmetry on the $16 s_{i} \kappa_{j}$ matrices are listed in Table I. Combining Eq. (E3) and the results in Table I, we obtain the effective theory on edge I in Fig. 7 with the general form 
TABLE I. The constraints of time reversal symmetry $(\Theta)$ and particle-hole symmetry $(\mathcal{P})$ on the $16 s_{i} \kappa_{j}$ matrices. In the table, we use $+(-)$ to label the matrices satisfying $\mathcal{U} s_{i} \kappa_{j} \mathcal{U}^{-1}=s_{i} \kappa_{j}\left(\mathcal{U} s_{i} \kappa_{j} \mathcal{U}^{-1}=-s_{i} \kappa_{j}\right)$, with $\mathcal{U}$ being $\Theta$ or $\mathcal{P}$.

\begin{tabular}{|c|c|c|c|c|c|c|c|c|c|c|c|c|c|c|c|c|}
\hline & $s_{0} \kappa_{0}$ & $s_{0} \kappa_{1}$ & $s_{0} \kappa_{2}$ & $s_{0} \kappa_{3}$ & $s_{1} \kappa_{0}$ & $s_{1} \kappa_{1}$ & $s_{1} \kappa_{2}$ & $s_{1} \kappa_{3}$ & $s_{2} \kappa_{0}$ & $s_{2} \kappa_{1}$ & $s_{2} \kappa_{2}$ & $s_{2} \kappa_{3}$ & $s_{3} \kappa_{0}$ & $s_{3} \kappa_{1}$ & $s_{3} \kappa_{2}$ & $s_{3} \kappa_{3}$ \\
\hline$\Theta$ & + & + & - & + & - & - & + & - & - & - & + & - & - & - & + & - \\
\hline $\mathcal{P}$ & + & - & - & - & - & + & + & + & - & + & + & + & - & + & + & + \\
\hline
\end{tabular}

$\mathcal{H}_{\mathrm{eff}, \mathrm{I}}=v k_{y} \sum_{i=1}^{3}\left(\alpha_{i} s_{i} \kappa_{1}+\beta_{i} s_{i} \kappa_{3}\right)+m\left(\alpha_{0} s_{0} \kappa_{1}+\beta_{0} s_{0} \kappa_{3}\right)$

where $v$ is the Fermi velocity, $m$ is the constant mass, and $\alpha$ and $\beta$ are the coefficients. We apply the mirror symmetry $\mathcal{M}_{y}$ to the above effective theory. To derive the matrix form of the mirror symmetry, one should keep in mind that (i) $\mathcal{M}_{y}$ satisfies the constraints in Eq. (E4) and it stabilizes two pairs of gapless modes on edge I [the constant mass in Eq. (E5) should be prohibited by mirror symmetry]; (ii) similar to the analysis in the above section, the gapless modes in the same mirror subspace always carry the same chirality, and the gapless modes with opposite mirror eigenvalue must carry opposite chirality (for a gapless mode on edge $\mathrm{I}$, its $\mathcal{M}_{y}$ eigenvalue is locked with its $\mathcal{C}$ eigenvalue, making the two symmetries satisfy $i \mathcal{M}_{y}=\mathcal{C}$ or $i \mathcal{M}_{y}=-\mathcal{C}$ ). Accordingly, the mirror symmetry can be chosen as $\mathcal{M}_{y}=i s_{0} \kappa_{2}$. The effective theory on edge I in Fig. 7 takes the form $\mathcal{H}_{\text {eff,I }}=v k_{y} \sum_{i=1}^{3}\left(\alpha_{i} s_{i} \kappa_{1}+\beta_{i} s_{i} \kappa_{3}\right)$. For simplicity, we take $\mathcal{H}_{\text {eff,I }}=v k_{y} s_{3} \kappa_{3}$ in the following (the other cases can be analyzed similarly).

Then, we bend edge I and make the bent edge evolve into edges II and III in Fig. 7 gradually. In this progress, the gapless modes on edge II (III) gain mass and are gapped out, as mirror symmetry cannot be maintained on each of the edges. Considering the symmetry constraints in Eq. (E3), we have the mass term as $m_{\mathrm{II} / \mathrm{III}} s_{0} \kappa_{1}$, with $m_{\mathrm{II} / \mathrm{III}}$

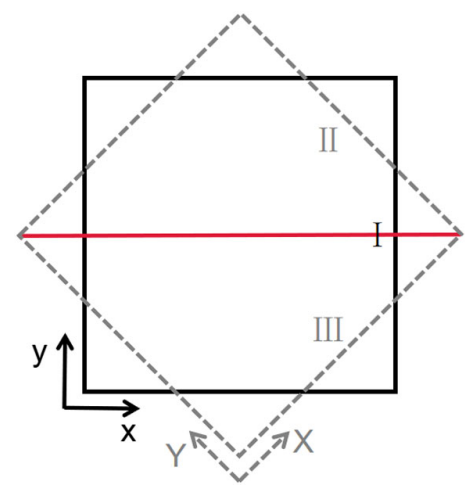

FIG. 7. Sketch of two different open-boundary conditions. The square in black solid lines shows the open boundaries in the (10) and (01) directions, while the square in gray dashed lines shows the open boundaries in the (11) and (11) directions. Mirror symmetry is implied by the red line in the figure. the mass term on edge II/III. Therefore, the effective theories on edges II andIII in Fig. 7 can be written as

$\mathcal{H}_{\text {eff,II }}=v k_{Y} s_{3} \kappa_{3}+m_{\mathrm{II}} s_{0} \kappa_{1}, \quad \mathcal{H}_{\text {eff,III }}=v k_{X} s_{3} \kappa_{3}+m_{\mathrm{III}} s_{0} \kappa_{1}$.

Moreover, since edges II andIII are related by the mirror reflection $\mathcal{M}_{y}$, the effective theories on the two edges satisfy $\mathcal{M}_{y} \mathcal{H}_{\text {eff,II }} \mathcal{M}_{y}^{-1}=\mathcal{H}_{\text {eff,III }}$ (notice that $\mathcal{M}_{\mathrm{y}} k_{X}=-k_{Y}$ ). Therefore, we have $m_{\text {II }}=-m_{\text {III }}$.

The theory in Eq. (E6) can be written in an instructive form by treating the gapless part as propagating modes along the edges of a finite size system, $\mathcal{H}_{\text {eff }}=v k s_{3} \kappa_{3}+$ $m_{r} s_{0} \kappa_{1}$ with $m_{r}$ changing sign at the intersection between the (11) and (1 $1 \overline{1})$ edges (assuming the intersection, i.e., the corner, at $r=0$, we have $\left.m_{r>0}=-m_{r<0}\right)$. In this form, $\mathcal{H}_{\text {eff }}$ is just a Dirac theory with a mass domain at $r=0$. It naturally leads to a pair of Majorana zero modes

$\left|\varphi_{1}\right\rangle=e^{-\int_{0}^{r}\left(m_{r^{\prime}} / v\right) d r^{\prime}}\left|s_{3}=1\right\rangle \otimes\left|\kappa_{2}=-1\right\rangle / \sqrt{2}$,
$\left|\varphi_{2}\right\rangle=e^{-\int_{0}^{r}\left(m_{r^{\prime}} / v\right) d r^{\prime}}\left|s_{3}=-1\right\rangle \otimes\left|\kappa_{2}=1\right\rangle / \sqrt{2}$,

where $v$ and $m_{r>0}$ are assumed to be positive and $\left|s_{3}= \pm 1\right\rangle$ $\left(\left|\kappa_{2}= \pm 1\right\rangle\right)$ is the eigenfunction of $s_{3}\left(\kappa_{2}\right)$ with eigenvalue \pm 1 . Obviously, the two Majorana zero modes in Eq. (E7) are time reversal partners, and they are both localized at $r=0$ (the corner).

Before finishing this part, it is worth emphasizing the following points. (i) The Majorana modes emerge robustly

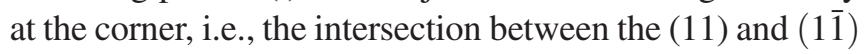
edges, since the mass domain at the corner is intrinsically guaranteed by the mirror symmetry $\mathcal{M}_{y}$. (ii) Though we focus on the corner at the intersection between the (11) and (11) edges, the analysis can be generalized to any corner respecting the mirror symmetry $\mathcal{M}_{y}$. (iii) As the whole system respects the space group $P 4 / \mathrm{nmm}$ where there exists fourfold rotational symmetry, the above analysis can be generalized to the corners related by fourfold rotational symmetry (corners respecting the mirror symmetry $\mathcal{M}_{x}$ ). (iv) The $\mathcal{Z}$ classification of the corner Majorana modes is lowered down to $\mathcal{Z}_{2}$ if we consider a corner which slightly breaks the mirror symmetry; i.e., there can be one single Majorana Kramers' pair at the corner which is protected by time reversal symmetry in this condition. 


\section{APPENDIX F: TOPOLOGICAL SUPERCONDUCTIVITY IN THE 3D LATTICES RESPECTING THE $P 4 / \mathrm{nmm}$ SYMMETRY GROUP}

In the main text, we generalize the topological superconductivity from the $2 \mathrm{D}$ case to $3 \mathrm{D}$ systems. Assuming no accidental nodes in the bulk superconducting spectrum, we find that there can exist three different kind of phases: a full-gap topological trivial phase, a full-gap second-order topological superconductor phase, and a second-order topological Dirac superconductor phase [58]. All the topological phases are protected by the mirror symmetries $\mathcal{M}_{x}$ and $\mathcal{M}_{y}$. Here, we specify the statement based on numerical simulations.

We stack the 2D lattice model in the main text along the $z$ direction and assume the 3D system weakly coupled along the $z$ direction. In a most simple condition, the system can be depicted by the following Hamiltonian:

$$
\mathcal{H}_{\mathrm{BdG}, 3 \mathrm{D}}=\left(\mathcal{H}_{0,3 \mathrm{D}}-\mu\right) \tau_{3}+\mathcal{H}_{\mathrm{sc}, 3 \mathrm{D}} \tau_{1}
$$

where $\mathcal{H}_{0,3 \mathrm{D}}=\mathcal{H}_{0}+2 t_{3}\left(\cos k_{z}-1\right) s_{0} \sigma_{0} \eta_{0}$ and $\mathcal{H}_{\mathrm{sc}, 3 \mathrm{D}}=$ $\mathcal{H}_{\mathrm{sc}}+2 \Delta_{3}\left(\cos k_{z}-1\right) s_{0} \sigma_{0} \eta_{0}$, with $\mathcal{H}_{0}$ and $\mathcal{H}_{\text {sc }}$ presented in the main text. In the system in Eq. (F1), we merely consider the $\mathrm{NN}$ intrasublattice intraorbital hopping $\left(t_{3}\right)$ and pairing $\left(\Delta_{3}\right)$ along the $z$ direction in addition to the lattice model in the main text. Notice that $\mathcal{H}_{\mathrm{BdG}, 3 \mathrm{D}}$ at $k_{z}=0$ has exactly the same form as the 2D lattice model in the main text. By tuning $t_{3}$ and $\Delta_{3}$ [with the other parameters the same as those in Fig. 3(a) in the main text], different 3D topological superconducting states can be realized. In the following, we focus on two cases.

(i) We set $t_{3}=0.15$ and $\Delta_{3}=0.03$. With these parameters, the two Fermi surfaces in Fig. 3(a) in the main text evolve into two quasi-2D concentric cylinders along the $k_{z}$ direction, and the pairing node of $\mathcal{H}_{\mathrm{sc}, 3 \mathrm{D}}$ sits between the two Fermi surfaces, as shown in Fig. 8(a). Therefore, in this condition the system is fully gapped with the pairing order on the two Fermi surfaces sign changed. Now we study the $\left\{M_{y} \mid \mathbf{0}\right\}$ protected winding number $w_{\pi}^{ \pm i}\left(k_{z}^{0}\right)$ on line $\left(k_{x}, \pi, k_{z}^{0}\right)$ for each fixed $k_{z}^{0}$. At $k_{z}=0$, as pointed out, $\mathcal{H}_{\mathrm{BdG}, 3 \mathrm{D}}$ is the same as the 2D lattice model in the main text, namely, $w_{\pi}^{+i}(0)=-w_{\pi}^{-i}(0)=2$. According to the Fermi surface condition in Fig. 3(a) and the analysis in the main text, it can be straightforwardly concluded that the winding numbers are all the same, namely, $w_{\pi}^{+i}\left(k_{z}^{0}\right)=-w_{\pi}^{-i}\left(k_{z}^{0}\right)=2$ for each fixed $k_{z}^{0}$ satisfying $-\pi \leq k_{z}^{0} \leq \pi$. Correspondingly, on the (100) surface where the mirror symmetry $\left\{M_{y} \mid \mathbf{0}\right\}$ is preserved, the winding number leads to fourfold degenerate zero-energy modes at each point on line $k_{y}=\pi$, i.e., the quasi-1D Dirac cones, as shown in Fig. 8(b); on the hinge between

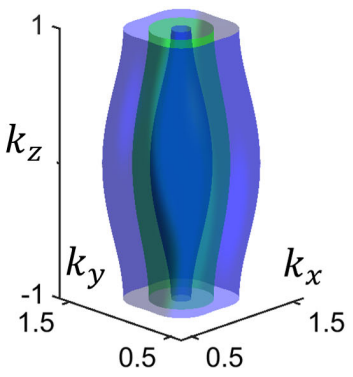

(a)

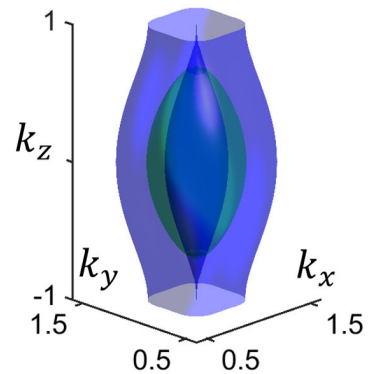

(d)

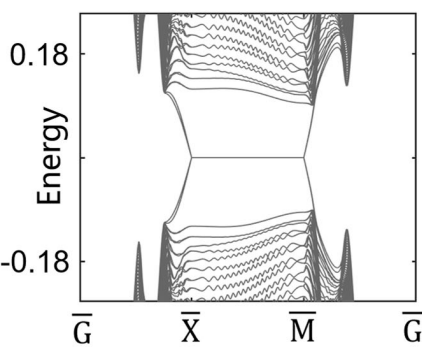

(b)

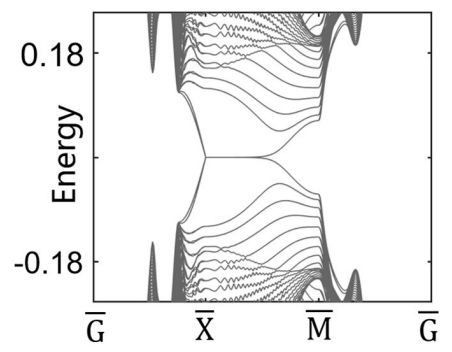

(e)

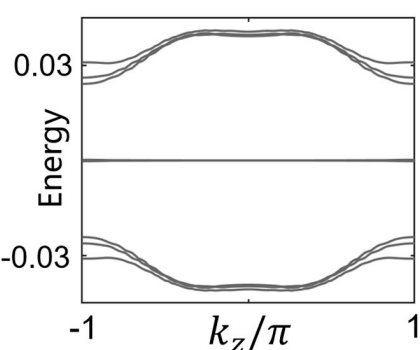

(c)

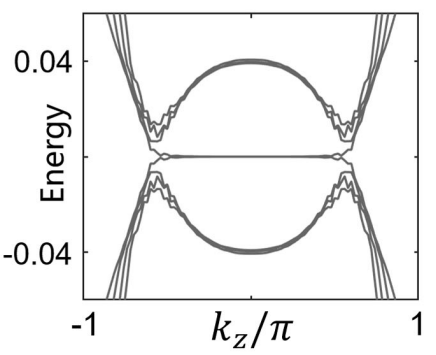

(f)

FIG. 8. (a) and (d) show the Fermi surfaces (blue) in the 3D system described by $\mathcal{H}_{0,3 \mathrm{D}}$ and the pairing nodes (green) for $\mathcal{H}_{\mathrm{sc}, 3 \mathrm{D}}$. The coordinate axis is in the unit of $\pi$. (b) and (e) show the superconducting surface modes along the high-symmetry lines on the (100) surface corresponding to the system described by $\mathcal{H}_{\mathrm{BdG}, 3 \mathrm{D}}$ in Eq. (F1). $\bar{G}, \bar{X}$, and $\bar{M}$ are the high-symmetry points in the surface Brillouin zone $\left(k_{y}, k_{z}\right)$, with $\bar{G}, \bar{X}$, and $\bar{M}$ being $(0,0),(\pi, 0)$, and $(\pi, \pi)$, respectively. (d) and (e) show the hinge modes corresponding to the system $\mathcal{H}_{\mathrm{BdG}, 3 \mathrm{D}}$, under open-boundary conditions in both the (110) and (110) directions. We take $t_{3}=0.15$ and $\Delta_{3}=0.03$ in (a) $-(\mathrm{c})$ and $t_{3}=0.2$ and $\Delta_{3}=0.07$ in (d)-(f). The other parameters are all the same as those in Fig. 3(a) in the main text. 
the (110) and (110) surfaces, the system supports twofold degenerate Majorana flat bands across the 1D Brillouin zone, as shown in Fig. 8(c).

(ii) In the second case, we adopt $t_{3}=0.2$ and $\Delta_{3}=$ 0.07. Compared to case (i), the larger $t_{3}$ and $\Delta_{3}$ make the system more dispersive along $k_{z}$, which can be seen from the Fermi surfaces and the pairing node shown in Fig. 8(d). Moreover, the pairing node intersects with the inner Fermi surface, leading to nodal lines around $k_{z}= \pm 0.65 \pi$ in the bulk superconducting gap as indicated in Fig. 8(d) (the nodal line can shrink to a nodal point by tuning parameters). Similar to the analysis in case (i), the mirror-symmetry-protected winding number is $w_{\pi}^{+i}\left(k_{z}^{0}\right)=-w_{\pi}^{-i}\left(k_{z}^{0}\right)=2$ for $-0.65 \pi<k_{z}^{0}<0.65 \pi$. For $\left|k_{z}^{0}\right|>0.65 \pi$, the superconducting pairing on the Fermi surfaces has no sign change, so we have $w_{\pi}^{+i}\left(k_{z}^{0}\right)=-w_{\pi}^{-i}\left(k_{z}^{0}\right)=0$. According to the above analysis, we immediately come to the following conclusions. The system must have zero-energy modes at each point satisfying $-0.65 \pi<k_{z}<$ $0.65 \pi$ on the $k_{y}=\pi$ line on the (100) surface as shown in Fig. 8(e) and Majorana flat bands for $-0.65 \pi<k_{z}<0.65 \pi$ on the hinge between the (110) and (110) surfaces as shown in Fig. 8(f).

\section{APPENDIX G: CALCULATIONS FOR THE IRON-SELENIDE SUPERCONDUCTORS}

In this part, we derive the topological superconductivity for iron chalcogenides. We consider the following Hamiltonian that captures the band structure of the monolayer FeSe:

$$
\mathcal{H}_{0}=\mathcal{H}_{\mathrm{TB}}+\mathcal{H}_{\mathrm{soc}}
$$

where $\mathcal{H}_{\mathrm{TB}}$ and $\mathcal{H}_{\mathrm{Soc}}$ are the tight-binding part and the spinorbit coupling part, respectively. For the tight-binding part, we adopt the Hamiltonian in Ref. [104], in which all five $d$ orbitals of the Fe atoms are taken into account, and utilize the parameters in Table II to fit the band structures of a single layer FeSe shown in Fig. 9(a). For the spin-orbit coupling part, we merely consider the atomic spin-orbit coupling of the $d$ orbitals, namely, $\mathcal{H}_{\mathrm{soc}}=\lambda \mathbf{L} \cdot \mathbf{s}$ with $\mathbf{L}$ and $\mathbf{s}$ the orbital and spin angular momentum, respectively. If we write the spin-orbit coupling as

$\mathcal{H}_{\mathrm{soc}}=\mathbf{d}_{\uparrow}^{\dagger} h_{\uparrow \uparrow} \mathbf{d}_{\uparrow}+\mathbf{d}_{\uparrow}^{\dagger} h_{\uparrow \downarrow} \mathbf{d}_{\downarrow}+\mathbf{d}_{\downarrow}^{\dagger} h_{\downarrow \uparrow} \mathbf{d}_{\uparrow}+\mathbf{d}_{\downarrow}^{\dagger} h_{\downarrow \downarrow} \mathbf{d}_{\downarrow}$

with $\mathbf{d}=\left(d_{x z}, d_{y z}, d_{x^{2}-y^{2}}, d_{x y}, d_{z^{2}}\right)^{T}$ as adopted in Table II, we have

$$
\begin{aligned}
h_{\uparrow \uparrow}= & \frac{\lambda}{2}\left(\begin{array}{ccccc}
0 & -i & 0 & 0 & 0 \\
i & 0 & 0 & 0 & 0 \\
0 & 0 & 0 & -2 i & 0 \\
0 & 0 & 2 i & 0 & 0 \\
0 & 0 & 0 & 0 & 0
\end{array}\right), \\
h_{\uparrow \downarrow}= & \frac{\lambda}{2}\left(\begin{array}{ccccc}
0 & 0 & 1 & -i & -\sqrt{3} \\
0 & 0 & i & 1 & \sqrt{3} i \\
-1 & -i & 0 & 0 & 0 \\
i & -1 & 0 & 0 & 0 \\
\sqrt{3} & -\sqrt{3} i & 0 & 0 & 0
\end{array}\right) .
\end{aligned}
$$

By applying the time reversal partners to $h_{\uparrow \uparrow}$ and $h_{\uparrow \downarrow}, h_{\downarrow \downarrow}$ and $h_{\downarrow \uparrow}$ can be obtained accordingly. It is worth pointing out that the atomic spin-orbit coupling combined with the lattice structure of the iron-based superconductors shown in Fig. 9(e) can result in effective Rashba-type spin-orbit coupling similar to the lattice model in the main text. Figure 9(b) shows the bands in the presence of spin-orbit coupling $(\lambda=40 \mathrm{meV})$. In the following, we set the chemical potential to be $\mu=118.3 \mathrm{meV}$ (the doping level

TABLE II. Hopping parameters for the monolayer FeSe. The on-site energies of the $d$ orbitals are $\epsilon_{1}=0.1754, \epsilon_{3}=-0.3576$, $\epsilon_{4}=0.0904$, and $\epsilon_{5}=-0.2776$. We adopt the same notations as those in Ref. [104], and all the parameters are in the unit of eV. Notice

\begin{tabular}{|c|c|c|c|c|c|c|c|c|}
\hline$t_{i}^{m n}$ & $i=x$ & $i=y$ & $i=x y$ & $i=x x$ & $i=y y$ & $i=x x y$ & $i=x y y$ & $i=x x y y$ \\
\hline$m n=11$ & -0.1514 & -0.4059 & 0.225 & 0.002 & -0.036 & -0.019 & 0.014 & 0.024 \\
\hline$m n=33$ & -0.4584 & & -0.070 & -0.013 & & & & 0.012 \\
\hline$m n=44$ & -0.0704 & & 0.012 & 0.002 & & 0.019 & & -0.024 \\
\hline$m n=55$ & & & 0.013 & -0.014 & & -0.006 & & -0.011 \\
\hline$m n=12$ & & & 0.103 & & & -0.011 & & 0.032 \\
\hline$m n=13$ & -0.473 & & -0.089 & & 0.011 & 0.018 & -0.006 & \\
\hline$m n=14$ & -0.2736 & & 0.053 & -0.001 & & -0.006 & & -0.009 \\
\hline$m n=15$ & -0.200 & & -0.130 & & 0.009 & 0.009 & 0.011 & -0.012 \\
\hline$m n=34$ & & & & & & 0.012 & & \\
\hline$m n=35$ & 0.401 & & & -0.023 & & 0.006 & & \\
\hline$m n=45$ & & & -0.113 & & & & & 0.011 \\
\hline
\end{tabular}
that according to Ref. [104] in the table $(1,2,3,4,5)$ correspond to the orbitals $\left(d_{x z}, d_{y z}, d_{x^{2}-y^{2}}, d_{x y}, d_{z^{2}}\right)$, and the $x$ direction is along the nearest $\mathrm{Fe} 1-\mathrm{Fe} 2$ bond in the monolayer FeSe shown in Fig. 9(e). 


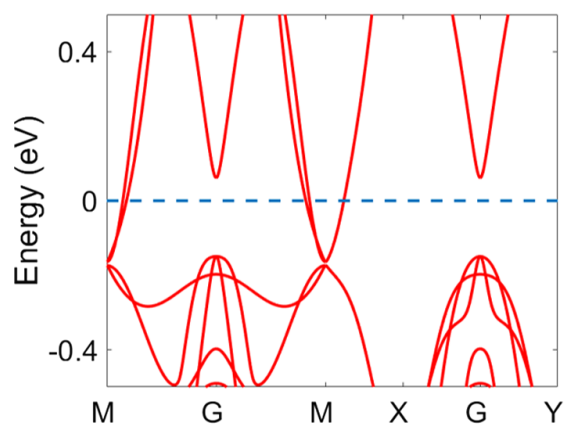

(a)

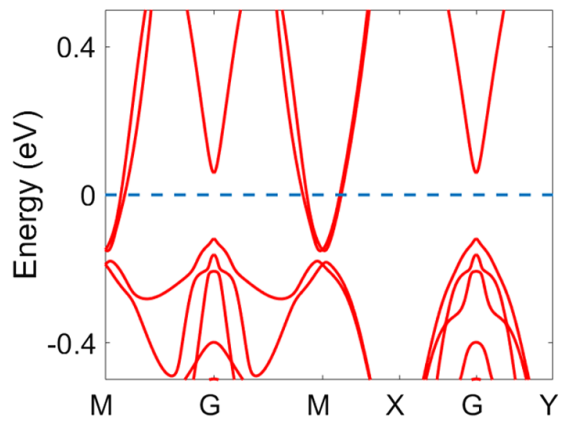

(b)

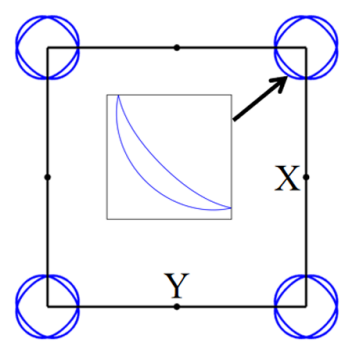

(c)

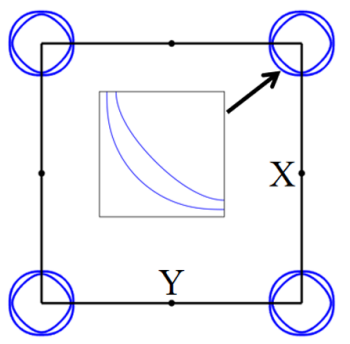

(d)

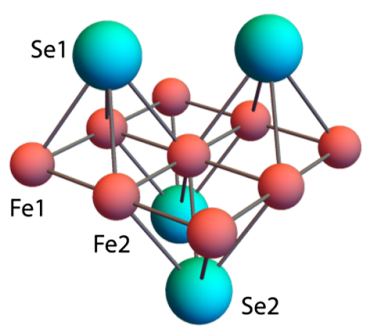

(e)

FIG. 9. (a) and (b) show the normal-state band structures of the monolayer FeSe in the absence and presence of spin-orbit coupling, respectively. The corresponding Fermi surfaces are shown in (c) and (d), with the insets showing the details. The chemical potential is set to be $\mu=118.3 \mathrm{meV}$, and the spin-orbit coupling $\lambda=40 \mathrm{meV}$ here. We also show the lattice structure of the monolayer FeSe in (e).

is about 0.09 electron per Fe), and the corresponding Fermi surfaces are presented in Figs. 9(c) and 9(d).

\section{Numerical simulation for the topological superconductivity in the iron-selenide superconductors}

Based on the genuine model in Eq. (G1), we calculate the edge modes in the superconducting state to verify possible topological superconductivity in iron-based superconductors. For the superconducting part, we design a general superconducting gap form which includes the on-site and NN intrasublattice intraorbital $s$-wave spin-singlet pairing [97], namely,

$$
\mathcal{H}_{\mathrm{sc}}=\Delta_{0}+2 \Delta_{1}\left(\cos k_{X}+\cos k_{Y}\right)
$$

where $k_{X}$ and $k_{Y}$ are defined in the two-Fe unit cell. This form can generate both conventional and sign-changed $s$ waves between inner and outer pockets. We consider the sign-changed $s$ wave to confirm its nontrivial topology. Taking $\Delta_{0}=-58 \mathrm{meV}$ and $\Delta_{1}=-16.7 \mathrm{meV}$, the superconducting state is the sign-changed $s$-wave pairing state with a minimum gap around $0.7 \mathrm{meV}$, as shown in Fig. 10(a).

With the above parameters, we calculate the edge modes on the (10) boundary shown in Fig. 10(b). There are two degenerate Majorana cones, which is consistent with the analysis in the main text. Therefore, we can conclude that the sign-changed $s$-wave state in iron-selenides is a secondorder topological superconductor.

\section{More analysis on the pairing states in the iron- selenide superconductors}

So far, we have shown that, in the iron-selenide superconductors with only two electron pockets near the Brillouin zone corner, sign-changed $s$-wave pairing corresponds to a second-order topological superconducting state. Here, we present more analysis on the pairing states (a)

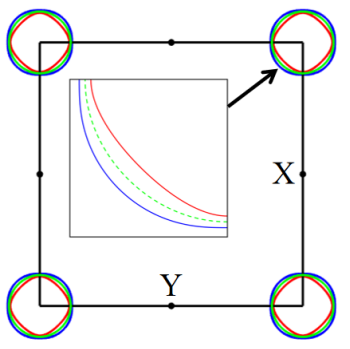

(b)

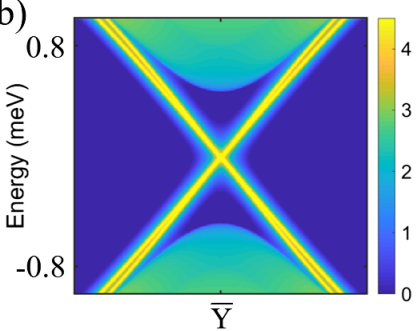

FIG. 10. (a) shows the sign-changed $s$-wave pairing simulated by the superconducting order in Eq. (G4), with the sign of the superconducting order indicated by red $(+)$ and blue $(-)$. The Fermi surfaces are in the same condition as those in Fig. 9(d), and the pairing nodes are labeled by the green color. (b) shows the edge modes around $\bar{Y}$ on the (10) boundary, corresponding to the superconducting state in (a). 


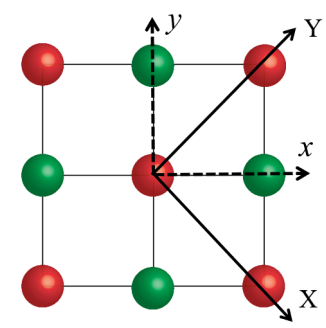

(a)

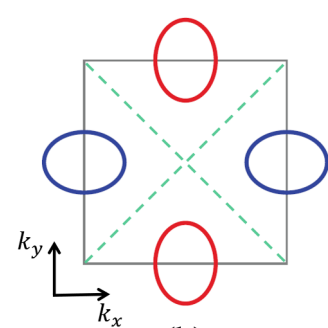

(b)

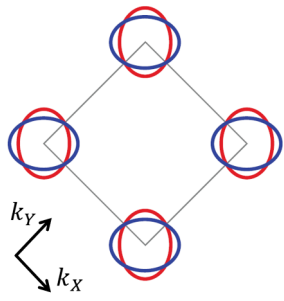

(c)

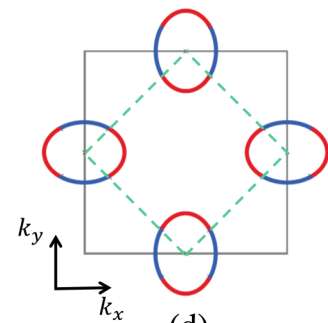

(d)

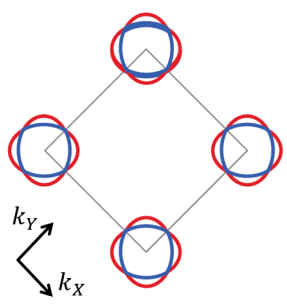

(e)

FIG. 11. (a) shows the sublattice structure of monolayer FeSe. (b)-(e) sketch the NN intersublattice pairing states in the absence of spin-orbit coupling, with the pairing sign on the Fermi surfaces indicated by the red and blue colors. (b) and (c) correspond to $d$-wave pairing and (d) and (e) to $s$-wave pairing. In the figures, (b) and (d) show the one-Fe-unit-cell case with the pairing nodes marked by the green dashed lines, and (c) and (e) show the two-Fe-unit-cell case.

in the iron-selenide superconductors, especially the pairing in the sublattice degree of freedom.

In the former part, in the numerical calculations intrasublattice $s$-wave pairing with the specific form $\mathcal{H}_{\mathrm{sc}}=$ $\Delta_{0}+2 \Delta_{1}\left(\cos k_{X}+\cos k_{Y}\right)$ is taken. One can also consider intersublattice pairing. However, the main problem for intersublattice $s$-wave pairing is that it always leads to the nodal superconducting state in iron-selenide superconductors. For instance, NN intersublattice $s$-wave pairing takes the form $\mathcal{H}_{\mathrm{sc}}^{\mathrm{NN}, s, 1}=2 \Delta_{\mathrm{NN}, s}\left(\cos k_{x}+\cos k_{y}\right) \eta_{0}$ in the one-Fe-unit-cell case, which vanishes along lines $k_{x}+k_{y}= \pm \pi \quad$ and $\quad k_{x}-k_{y}= \pm \pi, \quad$ and $\quad \mathcal{H}_{\mathrm{sc}}^{\mathrm{NN}, s, 2}=$ $4 \Delta_{\mathrm{NN}, S} \cos k_{X} / 2 \cos k_{Y} / 2 \eta_{1}$ in the two-Fe-unit-cell case, which vanishes along lines $k_{X}= \pm \pi$ and $k_{Y}= \pm \pi$, as illustrated in Figs. 11(d) and 11(e). Here, $\left(k_{x}, k_{y}\right)$ and $\left(k_{X}, k_{Y}\right)$ are defined in accordance with $(x, y)$ and $(X, Y)$ shown in Fig. 11(a), respectively. Therefore, to get the fullgap $s$-wave superconducting state, we have to consider intrasublattice pairing, such as the specific form in Eq. (G4), in the presence of spin-orbit coupling (finite spin-orbit coupling is necessary). It is worth pointing out that (i) substituting the pairing form in Eq. (G4) with longrange pairing and (ii) mixing intrasublattice pairing with intersublattice pairing do not change the conclusions in this work.

Besides $s$-wave pairing, in monolayer FeSe, $d$-wave pairing can also support the full-gap superconducting state. However, as pointed out in previous studies $[67,76]$, the $d$-wave state can be full gap only when the hybridization between the inner and outer Fermi surfaces shown in Fig. 9(c) is weak compared to the superconducting pairing strength. The requirement lies in the fact that, if the $d$-wave pairing is projected onto the band basis, in the strong hybridization condition the inner and outer Fermi surfaces split largely and only the intraband pairing matters. The intraband pairing must have nodes in the $d$-wave state. However, if the split of the two Fermi surfaces is small, besides intraband pairing, interband pairing also plays an important role, and it can make the $d$-wave superconductivity full gap. Considering that the inner and outer Fermi surfaces in monolayer FeSe hybridize thoroughly due to spin-orbit coupling as indicated in Figs. 9(a)-9(d), we immediately conclude that the $d$-wave state can be full gap only when the spin-orbit coupling is weak (including the vanishing case). Specifically, in monolayer FeSe, to get full-gap $d$-wave superconductivity, we need to consider intersublattice pairing (it can be checked that intrasublattice $d$-wave states are always nodal). We take NN intersublattice $d$-wave pairing as an example, which has the form $\mathcal{H}_{\mathrm{sc}}^{\mathrm{NN}, d, 1}=2 \Delta_{\mathrm{NN}, s}\left(\cos k_{x}-\cos k_{y}\right) \eta_{0}$ in the one-Fe-unitcell case and $\mathcal{H}_{\mathrm{sc}}^{\mathrm{NN}, d, 2}=4 \Delta_{\mathrm{NN}, s} \sin k_{X} / 2 \sin k_{Y} / 2 \eta_{1}$ in the two-Fe-unit-cell case, and the superconducting state is sketched in Figs. 11(b) and 11(c). This state is full gap (and topologically trivial) in the absence of spin-orbit coupling and evolves into the nodal state as the spin-orbit coupling becomes stronger (this nodal state may carry topologically nontrivial properties).

Before finishing this part, it is worth mentioning that, in addition to the sublattice degree of freedom, the pairing in the orbital degree is also an important issue in iron-based superconductors. However, we point out that these issues do not change the conclusion in the work, since the topological state is decided by the superconducting pairing on the Fermi surfaces as demonstrated in the main text.

\section{Effective Rashba spin-orbit coupling in iron-selenide superconductors}

As mentioned, in iron-based superconductors, atomic spin-orbit coupling combined with lattice structure can lead to effective Rashba-type spin-orbit coupling similar to the lattice model in the main text. In monolayer FeSe, the inversion center is located at the middle of the nearest $\mathrm{Fe} 1-\mathrm{Fe} 2$ bond, and within the $\mathrm{Fe} 1(\mathrm{Fe} 2)$ sublattice there is no inversion symmetry, as indicated in Fig. 9(e). Therefore, Rashba spin-orbit coupling is prohibited between the nearest $\mathrm{Fe} 1$ and $\mathrm{Fe} 2$ sites, and we need to consider it between the nearest $\mathrm{Fe} 1(\mathrm{Fe} 2)$ and $\mathrm{Fe} 1(\mathrm{Fe} 2)$ atoms, i.e., the intrasublattice nearest neighbors.

In the genuine model in Eq. (G1), the bands near the Fermi energy are mainly contributed by the $d_{x z}, d_{y z}$, and 


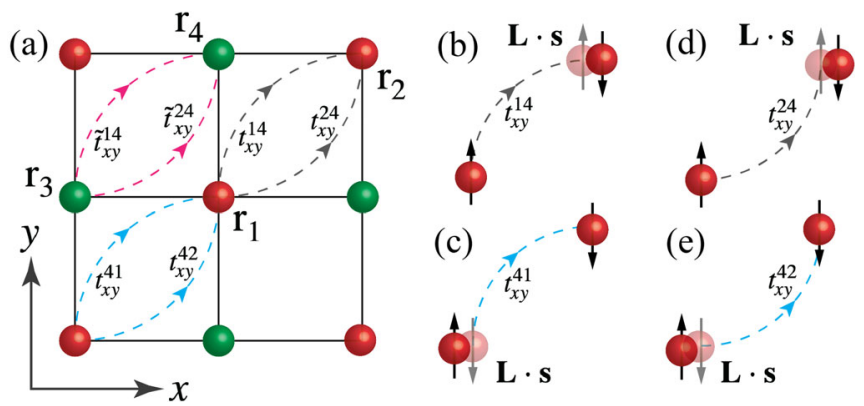

FIG. 12. (a) shows the NN intrasublattice hoppings (dashed lines) between the $d_{x z / y z}$ and $d_{x y}$ orbitals in iron-based superconductors, with the hopping parameters $t_{i}^{m n}$ shown in Table II. The hoppings in gray and blue occur within the Fe1 sublattice, and the hoppings in magenta occur within the $\mathrm{Fe} 2$ sublattice. Notice that the $x / y$ direction in (a) has a difference of $\pi / 4$ rotation with the primitive lattice vectors. (b)-(e) show the four secondorder processes which can induce the Rashba term $\lambda_{R} d_{x y, \uparrow}^{\dagger}\left(\mathbf{r}_{1}\right) d_{x y, \downarrow}\left(\mathbf{r}_{2}\right)$ in (a). The second-order processes are intermediated by the $d_{x z / y z}$ orbital [the half-transparent red ball which represents $d_{x z, \uparrow}, d_{x z, \downarrow}, d_{y z, \uparrow}$, and $d_{y z, \downarrow}$ in (b), (c), (d), and (e), respectively]. The dashed lines in (b)-(e) have the same meaning as those in (a).

$d_{x y}$ orbitals [104]. Here, we take the $d_{x y}$ orbital between the intrasublattice nearest neighbors, for instance, to estimate the strength of the effective Rashba spin-orbit coupling in monolayer FeSe. Specifically, we consider the two $d_{x y}$ orbitals at $\mathbf{r}_{1}$ and $\mathbf{r}_{2}$ in Fig. 12(a). The effective Rashba spin-orbit coupling between these two orbitals should take the form $\mathcal{H}_{R}=\lambda_{R} d_{x y, \uparrow}^{\dagger}\left(\mathbf{r}_{1}\right) d_{x y, \downarrow}\left(\mathbf{r}_{2}\right)-\lambda_{R}^{*} d_{x y, \downarrow}^{\dagger}\left(\mathbf{r}_{1}\right) d_{x y, \uparrow}\left(\mathbf{r}_{1}\right)+$ H.c., which can be generated by the high-order processes in the genuine model in Eq. (G1). With a careful check, it can be found that up to second order the term $\lambda_{R} d_{x y, \uparrow}^{\dagger}\left(\mathbf{r}_{1}\right) d_{x y, \downarrow}\left(\mathbf{r}_{2}\right)$ can be induced by the four processes listed in Figs. 12(b)-12(e). These second-order processes are intermediated by the $d_{x z / y z}$ orbital, and in each of the second-order processes it includes direct hopping and atomic spin-orbit coupling between the $d_{x y}$ and $d_{x z / y z}$ orbitals. By taking the second-order processes in Figs. 12(b)12(e) and their reverse processes into account, we construct a space $\left[d_{x y, \uparrow}\left(\mathbf{r}_{1}\right), d_{x y, \downarrow}\left(\mathbf{r}_{2}\right), d_{x z, \downarrow}\left(\mathbf{r}_{1}\right), d_{y z, \downarrow}\left(\mathbf{r}_{1}\right), d_{x z, \uparrow}\left(\mathbf{r}_{2}\right)\right.$, $\left.d_{y z, \uparrow}\left(\mathbf{r}_{2}\right)\right]$. The space can be described by the following Hamiltonian:

$H=\left(\begin{array}{cc}H_{0} & T_{01} \\ T_{10} & H_{1}\end{array}\right), \quad T_{01}=\frac{1}{2}\left(\begin{array}{cccc}i \lambda & -\lambda & 2 t_{x y}^{14} & 2 t_{x y}^{24} \\ \left(2 t_{x y}^{41}\right)^{*} & \left(2 t_{x y}^{42}\right)^{*} & i \lambda & \lambda\end{array}\right)$,

with $H_{0}$ and $H_{1}$ corresponding to the subspace $\left[d_{x y, \uparrow}\left(\mathbf{r}_{1}\right)\right.$, $\left.d_{x y, \downarrow}\left(\mathbf{r}_{2}\right)\right] \quad$ and $\left[d_{x z, \downarrow}\left(\mathbf{r}_{1}\right), d_{y z, \downarrow}\left(\mathbf{r}_{1}\right), d_{x z, \uparrow}\left(\mathbf{r}_{2}\right), d_{y z, \uparrow}\left(\mathbf{r}_{2}\right)\right]$, respectively. In the above equation, $T_{10}=T_{01}^{\prime}, H_{0}=\epsilon_{4}$, and $H_{1}=\epsilon_{1}$ with $\epsilon_{1 / 4}$ presented in Table II. Considering the constraint of $C_{2 z}$ symmetry, the hopping parameters satisfy $-t_{x y}^{14}=-t_{x y}^{24}=t_{x y}^{42}=t_{x y}^{41}$. Integrating the $d_{x z / y z}$ orbitals out, we obtain the effective Hamiltonian for subspace $\left[d_{x y, \uparrow}\left(\mathbf{r}_{1}\right), d_{x y, \downarrow}\left(\mathbf{r}_{2}\right)\right] \quad$ as $\quad \tilde{H}_{0}=H_{0}-T_{01}\left(\epsilon_{4}-H_{1}\right)^{-1} T_{10}$. The off-diagonal terms in $\tilde{H}_{0}$ just correspond to the effective Rashba spin-orbit coupling $\lambda_{R} d_{x y, \uparrow}^{\dagger}\left(\mathbf{r}_{1}\right) d_{x y, \downarrow}\left(\mathbf{r}_{2}\right)+$ H.c., which gives $\lambda_{R}=-\lambda t_{x y}^{14}(1-i) /\left(\epsilon_{4}-\epsilon_{1}\right)$ with $\lambda$ the strength of the atomic spin-orbit coupling of the $d$ orbitals in ironbased superconductors.

Similarly, if we consider effective Rashba spin-orbit coupling between the two $d_{x y}$ orbitals at $\mathbf{r}_{3}$ and $\mathbf{r}_{4}$ in Fig. 12(a) with the form $\lambda_{R}^{\prime} d_{x y, \uparrow}^{\dagger}\left(\mathbf{r}_{3}\right) d_{x y, \downarrow}\left(\mathbf{r}_{4}\right)$, the strength can be calculated as $\lambda_{R}^{\prime}=-\lambda \tilde{t}_{x y}^{14}(1-i) /\left(\epsilon_{4}-\epsilon_{1}\right)$. Moreover, the symmetry group of iron-based superconductors requires $\tilde{t}_{x y}^{14}=-t_{x y}^{14}$, since the $\mathrm{Fe} 1$ and $\mathrm{Fe} 2$ sublattices are related by the glide mirror symmetry $\left\{M_{z} \mid \tau_{\mathbf{0}}\right\}$ as indicated in Fig. 9(e) (the $d_{x y}$ orbital is even under $M_{z}$, while $d_{x z / y z}$ is odd under $M_{z}$ ). Therefore, we have $\lambda_{R}^{\prime}=-\lambda_{R}$. Namely, the two sublattices experience opposite Rashba spin-orbit coupling, i.e., the opposite intrinsic polarization, which is consistent with the lattice model in the main text.

Based on the above estimation and the parameters in Table II, we estimate $\left|\lambda_{R}\right| \sim 0.88 \lambda$, which can be several tens of $\mathrm{meV}$ in monolayer FeSe. Moreover, it is worth pointing out that, in iron-based superconductors, effective Rashba spin-orbit coupling decides the band split along the $M-X$ line shown in Figs. 9(a)-G1(d). It can be verified by the fact that, for the bands described by the Hamiltonian in Eq. (G1), the spin-flip (spin-nonflip) part of the atomic spin-orbit coupling in Eq. (G2) can (cannot) split the bands along the $M-X$ line. This is consistent with the above analysis that effective Rashba spin-orbit coupling is induced from spin-flip spin-orbit coupling between the $d$ orbitals. In fact, in LiFeAs it has been identified that the band split along the $M-X$ line is about $10 \mathrm{meV}$ [110], which is consistent with our estimation.

\section{APPENDIX H: CENTROSYMMETRIC SPACE GROUPS WITH SIMILAR STRUCTURE WITH P4/nmm}

In the theory presented in the main text, the special group structure, i.e., inversion symmetry and mirror symmetry defined on nonequivalent sites, plays an essential role. Here, based on a query of all 230 space groups, we find that among the 92 centrosymmetric space groups there are 61 space groups, in each of which there is at least one point group operation defined at the nonequivalent site with the inversion center. According to our theory, in the $A_{1 g}$-pairing centrosymmetric superconductors respecting these space groups, topological superconductivity can be expected, though more detailed analysis needs to be carried out. We list these space groups (group number) as follows. 
(i) the monoclinic space group: $11,13,15$;

(ii) the orthorhombic space group: $48-54,56,57,59$, $60,62,63,66-68,70,74$;

(iii) the tetragonal space group: 84-86, 88, 124-127, 129-138, 140-142;

(iv) the trigonal space group: $163,165,167$;

(v) the hexagonal space group: 176, 192-194;

(vi) the cubic space group: 201, 203, 205, 206, 222-224, 226-228, 230.

[1] C. Nayak, S. H. Simon, A. Stern, M. Freedman, and S. D. Sarma, Non-Abelian Anyons and Topological Quantum Computation, Rev. Mod. Phys. 80, 1083 (2008).

[2] M.Z. Hasan and C. L. Kane, Colloquium: Topological Insulators, Rev. Mod. Phys. 82, 3045 (2010).

[3] X.-L. Qi and S.-C. Zhang, Topological Insulators and Superconductors, Rev. Mod. Phys. 83, 1057 (2011).

[4] C.-K. Chiu, J. C. Y. Teo, A. P. Schnyder, and S. Ryu, Classification of Topological Quantum Matter with Symmetries, Rev. Mod. Phys. 88, 035005 (2016).

[5] A. Y. Kitaev, Unpaired Majorana Fermions in Quantum Wires, Phys. Usp. 44, 131 (2001).

[6] J. Alicea, New Directions in the Pursuit of Majorana Fermions in Solid State Systems, Rep. Prog. Phys. 75, 076501 (2012).

[7] N. Hao and J. P. Hu, Topological Quantum States of Matter in Iron-Based Superconductors: From Concept to Material Realization, Natl. Sci. Rev. 6, 213 (2019).

[8] X. X. Wu, R.-X. Zhang, G. Xu, J. P. Hu, and C.-X. Liu, In the Pursuit of Majorana Modes in Iron-Based High- $\mathrm{T}_{\mathrm{c}}$ Superconductors, arXiv:2005.03603.

[9] L. Fu and C. L. Kane, Superconducting Proximity Effect and Majorana Fermions at the Surface of a Topological Insulator, Phys. Rev. Lett. 100, 096407 (2008).

[10] J. D. Sau, R. M. Lutchyn, S. Tewari, and S. D. Sarma, Generic New Platform for Topological Quantum Computation Using Semiconductor Heterostructures, Phys. Rev. Lett. 104, 040502 (2010).

[11] R. M. Lutchyn, J. D. Sau, and S. D. Sarma, Majorana Fermions and a Topological Phase Transition in Semiconductor-Superconductor Heterostructures, Phys. Rev. Lett. 105, 077001 (2010).

[12] Y. Oreg, G. Refael, and F. von Oppen, Helical Liquids and Majorana Bound States in Quantum Wires, Phys. Rev. Lett. 105, 177002 (2010).

[13] M.Wimmer, A. R. Akhmerov, M. V. Medvedyeva, J. Tworzydlo, and C. W. J. Beenakker, Majorana Bound States without Vortices in Topological Superconductors with Electrostatic Defects, Phys. Rev. Lett. 105, 046803 (2010).

[14] J. C. Y. Teo and C. L. Kane, Topological Defects and Gapless Modes in Insulators and Superconductors, Phys. Rev. B 82, 115120 (2010).

[15] P. Hosur, P. Ghaemi, R. S. K. Mong, and A. Vishwanath, Majorana Modes at the Ends of Superconductor Vortices in Doped Topological Insulators, Phys. Rev. Lett. 107, 097001 (2011).
[16] J. C. Y. Teo and T. L. Hughes, Existence of MajoranaFermion Bound States on Disclinations and the Classification of Topological Crystalline Superconductors in Two Dimensions, Phys. Rev. Lett. 111, 047006 (2013).

[17] F. Zhang, C. L. Kane, and E. J. Mele, Time-ReversalInvariant Topological Superconductivity and Majorana Kramers Pairs, Phys. Rev. Lett. 111, 056402 (2013).

[18] F. Pientka, L. I. Glazman, and F. von Oppen, Topological Superconducting Phase in Helical Shiba Chains, Phys. Rev. B 88, 155420 (2013).

[19] J. D. Sau and P. M. R. Brydon, Bound States of a Ferromagnetic Wire in a Superconductor, Phys. Rev. Lett. 115, 127003 (2015).

[20] J. Li, T. Neupert, B. A. Bernevig, and A. Yazdani, Manipulating Majorana Zero Modes on Atomic Rings with an External Magnetic Field, Nat. Commun. 7, 10395 (2016).

[21] G. Xu, B. Lian, P.Z. Tang, X.-L. Qi, and S.-C. Zhang, Topological Superconductivity on the Surface of Fe-Based Superconductors, Phys. Rev. Lett. 117, 047001 (2016).

[22] G. Yang, P. Stano, J. Klinovaja, and D. Loss, Majorana Bound States in Magnetic Skyrmions, Phys. Rev. B 93, 224505 (2016).

[23] K. Jiang, X. Dai, and Z. Q. Wang, Quantum Anomalous Vortex and Majorana Zero Mode in Iron-Based Superconductor Fe(Te,Se), Phys. Rev. X 9, 011033 (2019).

[24] M. F. Lapa, M. Cheng, and Y.X. Wang, SymmetryProtected Gates of Majorana Qubits in a High- $\mathrm{T}_{\mathrm{c}}$ Superconductor Platform, arXiv:2103.03893.

[25] S. Sasaki, M. Kriener, K. Segawa, K. Yada, Y. Tanaka, M. Sato, and Y. Ando, Topological Superconductivity in $\mathrm{Cu}_{x} \mathrm{Bi}_{2} \mathrm{Se}_{3}$, Phys. Rev. Lett. 107, 217001 (2011).

[26] A. Das, Y. Ronen, Y. Most, Y. Oreg, M. Heiblum, and H. Shtrikman, Zero-Bias Peaks and Splitting in an Al-InAs Nanowire Topological Superconductor as a Signature of Majorana Fermions, Nat. Phys. 8, 887 (2012).

[27] S. Nadj-Perge, I. K. Drozdov, J. Li, H. Chen, S. Jeon, J. Seo, A. H. MacDonald, B. A. Bernevig, and A. Yazdani, Observation of Majorana Fermions in Ferromagnetic Atomic Chains on a Superconductor, Science 346, 602 (2014).

[28] J.-P. Xu, M.-X. Wang, Z. L. Liu, J.-F. Ge, X. Yang, C. Liu, Z. A. Xu, D. Guan, C. L. Gao, D. Qian, Y. Liu, Q.-H. Wang, F.-C. Zhang, Q.-K. Xue, and J.-F. Jia, Experimental Detection of a Majorana Mode in the Core of a Magnetic Vortex inside a Topological Insulator-Superconductor $\mathrm{Bi}_{2} \mathrm{Te}_{3} / \mathrm{NbSe}_{2}$ Heterostructure, Phys. Rev. Lett. 114, 017001 (2015).

[29] D. F. Wang, L. Y. Kong, P. Fan, H. Chen, S. Y. Zhu, W. Y. Liu, L. Cao, Y. J. Sun, S. X. Du, J. Schneeloch, R. D. Zhong, G. D. Gu, L. Fu, H. Ding, and H.-J. Gao, Evidence for Majorana Bound States in an Iron-Based Superconductor, Science 362, 333 (2018).

[30] Q. Liu, C. Chen, T. Zhang, R. Peng, Y.-J. Yan, C.-H.-P. Wen, X. Lou, Y.-L. Huang, J.-P. Tian, X.-L. Dong, G.-W.Wang, W.-C. Bao, Q.-H. Wang, Z.-P. Yin, Z.-X. Zhao, and D.-L. Feng, Robust and Clean Majorana Zero Mode in the Vortex Core of High-Temperature Superconductor $\left(\mathrm{Li}_{0.84} \mathrm{Fe}_{0.16}\right)$ OHFeSe, Phys. Rev. X 8, 041056 (2018). 
[31] L. Y. Kong, S. Y. Zhu, M. Papaj, H. Chen, L. Cao, H. Isobe, Y. Q. Xing, W. Y. Liu, D. F. Wang, P. Fan, Y. J. Sun, S. X. Du, J. Schneeloch, R. D. Zhong, G. D. Gu, L. Fu, H.-J. Gao, and H. Ding, Half-Integer Level Shift of Vortex Bound States in an Iron-Based Superconductor, Nat. Phys. 15, 1181 (2019).

[32] T. Machida, Y. Sun, S. Pyon, S. Takeda, Y. Kohsaka, T. Hanaguri, T. Sasagawa, and T. Tamegai, Zero-Energy Vortex Bound State in the Superconducting Topological Surface State of $\mathrm{Fe}(\mathrm{Se}, \mathrm{Te})$, Nat. Mater. 18, 811 (2019).

[33] X. Y. Chen, M. Y. Chen, W. Duan, X. Y. Zhu, H. Yang, and H.-H. Wen, Observation and Characterization of the Zero Energy Conductance Peak in the Vortex Core State of $\mathrm{FeTe}_{0.55} \mathrm{Se}_{0.45}$, arXiv:1909.01686.

[34] L. Y. Kong, L. Cao, S. Y. Zhu, M. Papaj, G. Y. Dai, G. Li, P. Fan, W. Y. Liu, F. Z. Yang, X. C. Wang, S. X. Du, C. Q. Jin, L. Fu, H.-J. Gao, and H. Ding, Tunable Vortex Majorana Zero Modes in LiFeAs Superconductor, Nat. Commun. 12, 4146 (2021).

[35] Z. Y. Wang, J. O. Rodriguez, L. Jiao, S. Howard, M. Graham, G. D. Gu, T. L. Hughes, D. K. Morr, and V. Madhavan, Evidence for Dispersing 1D Majorana Channels in an Iron-Based Superconductor, Science 367, 104 (2020).

[36] S. Vaitiekenas, G. W. Winkler, B. van Heck, T. Karzig, M.-T. Deng, K. Flensberg, L. I. Glazman, C. Nayak, P. Krogstrup, R. M. Lutchyn, and C. M. Marcus, FluxInduced Topological Superconductivity in Full-Shell Nanowires, Science 367, 6485 (2020).

[37] Y. Ando and L. Fu, Topological Crystalline Insulators and Topological Superconductors: From Concepts to Materials, Annu. Rev. Condens. Matter Phys. 6, 361 (2015).

[38] L. Fu, Topological Crystalline Insulators, Phys. Rev. Lett. 106, 106802 (2011).

[39] T. H. Hsieh, H. Lin, J. W. Liu, W. H. Duan, A. Bansil, and L. Fu, Topological Crystalline Insulators in the SnTe Material Class, Nat. Commun. 3, 982 (2012).

[40] Y. Tanaka, Z. Ren, T. Sato, K. Nakayama, S. Souma, T. Takahashi, K. Segawa, and Y. Ando, Experimental Realization of a Topological Crystalline Insulator in SnTe, Nat. Phys. 8, 800 (2012).

[41] B. Bradlyn, L. Elcoro, J. Cano, M. Vergniory, Z. J. Wang, C. Felser, M. Aroyo, and B. A. Bernevig, Topological Quantum Chemistry, Nature (London) 547, 298 (2017).

[42] H. C. Po, A. Vishwanath, and H. Watanabe, SymmetryBased Indicators of Band Topology in the 230 Space Groups, Nat. Commun. 8, 50 (2017).

[43] J. Kruthoff, J. de Boer, J. van Wezel, C. L. Kane, and R.-J. Slager, Topological Classification of Crystalline Insulators through Band Structure Combinatorics, Phys. Rev. X 7, 041069 (2017).

[44] Z. D. Song, T. T. Zhang, Z. Fang, and C. Fang, Quantitative Mappings between Symmetry and Topology in Solids, Nat. Commun. 9, 3530 (2018).

[45] E. Khalaf, H. C. Po, A. Vishwanath, and H. Watanabe, Symmetry Indicators and Anomalous Surface States of Topological Crystalline Insulators, Phys. Rev. X 8, 031070 (2018).
[46] T. T. Zhang, Y. Jiang, Z. D. Song, H. Huang, Y. Q. He, Z. Fang, H. M. Weng, and C. Fang, Catalogue of Topological Electronic Materials, Nature (London) 566, 475 (2019).

[47] F. Tang, H. C. Po, A. Vishwanath, and X. G. Wan, Efficient Topological Materials Discovery Using Symmetry Indicators, Nat. Phys. 15, 470 (2019).

[48] Y. Ueno, A. Yamakage, Y. Tanaka, and M. Sato, SymmetryProtected Majorana Fermions in Topological Crystalline Superconductors: Theory and Application to $\mathrm{Sr}_{2} \mathrm{RuO}_{4}$, Phys. Rev. Lett. 111, 087002 (2013).

[49] Y. Tsutsumi, M. Ishikawa, T. Kawakami, T. Mizushima, M. Sato, M. Ichioka, and K. Machida, $\mathrm{UPt}_{3}$ as a Topological Crystalline Superconductor, J. Phys. Soc. Jpn. 82, 113707 (2013).

[50] F. Zhang, C. L. Kane, and E. J. Mele, Topological Mirror Superconductivity, Phys. Rev. Lett. 111, 056403 (2013).

[51] C. Fang, M. J. Gilbert, and B. A. Bernevig, New Class of Topological Superconductors Protected by Magnetic Group Symmetries, Phys. Rev. Lett. 112, 106401 (2014).

[52] S. Kobayashi and M. Sato, Topological Superconductivity in Dirac Semimetals, Phys. Rev. Lett. 115, 187001 (2015).

[53] C. Fang, B. A. Bernevig, and M. J. Gilbert, Topological Crystalline Superconductors with Linearly and Projectively Represented $C_{n}$ Symmetry, arXiv:1701.01944.

[54] R.-X. Zhang and C.-X. Liu, Crystalline SymmetryProtected Majorana Mode in Number-Conserving Dirac Semimetal Nanowires, Phys. Rev. Lett. 120, 156802 (2018).

[55] S. S. Qin, L. H. Hu, C. C. Le, J. F. Zeng, F.-C. Zhang, C. Fang, and J.P. Hu, Quasi-1D Topological Nodal Vortex Line Phase in Doped Superconducting 3D Dirac Semimetals, Phys. Rev. Lett. 123, 027003 (2019).

[56] E. J. König and P. Coleman, Crystalline-Symmetry-Protected Helical Majorana Modes in the Iron Pnictides, Phys. Rev. Lett. 122, 207001 (2019).

[57] S. S. Qin, L. H. Hu, X.X. Wu, X. Dai, C. Fang, F.-C. Zhang, and J. P. Hu, Topological Vortex Phase Transitions in Iron-Based Superconductors, Sci. Bull. 64, 1207 (2019).

[58] R.-X. Zhang, Y.-T. Hsu, and S. D. Sarma, Higher-Order Topological Dirac Superconductors, Phys. Rev. B 102, 094503 (2020).

[59] Q.-Z. Wang and C.-X. Liu, Topological Nonsymmorphic Crystalline Superconductors, Phys. Rev. B 93, 020505(R) (2016).

[60] K. Shiozaki, M. Sato, and K. Gomi, Topology of Nonsymmorphic Crystalline Insulators and Superconductors, Phys. Rev. B 93, 195413 (2016).

[61] A. Daido, T. Yoshida, and Y. Yanase, $\mathbb{Z}_{4}$ Topological Superconductivity in UCoGe, Phys. Rev. Lett. 122, 227001 (2019).

[62] A. P. Schnyder, S. Ryu, A. Furusaki, and A. W. W. Ludwig, Classification of Topological Insulators and Superconductors in Three Spatial Dimensions, Phys. Rev. B 78, 195125 (2008).

[63] S. Ryu, A. P. Schnyder, A. Furusaki, and A. W. W. Ludwig, Topological Insulators and Superconductors: Tenfold Way and Dimensional Hierarchy, New J. Phys. 12, 065010 (2010). 
[64] X.-L. Qi, T. L. Hughes, and S.-C. Zhang, Topological Invariants for the Fermi Surface of a Time-ReversalInvariant Superconductor, Phys. Rev. B 81, 134508 (2010).

[65] S. Ono, H. C. Po, and H. Watanabe, Refined Symmetry Indicators for Topological Superconductors in All Space Groups, Sci. Adv. 6, eaaz8367 (2020).

[66] K. Shiozaki, The Classification of Surface States of Topological Insulators and Superconductors with Magnetic Point Group Symmetry, arXiv:1907.09354.

[67] I. I. Mazin, Symmetry Analysis of Possible Superconducting States in $\mathrm{K}_{x} \mathrm{Fe}_{y} \mathrm{Se}_{2}$ Superconductors, Phys. Rev. B 84, 024529 (2011).

[68] P. J. Hirschfeld, M. M. Korshunov, and I. I. Mazin, Gap Symmetry and Structure of Fe-Based Superconductors, Rep. Prog. Phys. 74, 124508 (2011).

[69] X. X. Wu, W. A. Benalcazar, Y. X. Li, R. Thomale, C.-X. Liu, and J. P. Hu, Boundary-Obstructed Topological High$\mathrm{T}_{c}$ Superconductivity in Iron Pnictides, Phys. Rev. X 10, 041014 (2020).

[70] J. Hu, Iron-Based Superconductors as Odd-Parity Superconductors, Phys. Rev. X 3, 031004 (2013).

[71] X. W. Zhang, Q. H. Liu, J.-W. Luo, A. J. Freeman, and A. Zunger, Hidden Spin Polarization in Inversion-Symmetric Bulk Crystals, Nat. Phys. 10, 387 (2014).

[72] S.-L. Wu, K. Sumida, K. Miyamoto, K. Taguchi, T. Yoshikawa, A. Kimura, Y. Ueda, M. Arita, M. Nagao, S. Watauchi, I. Tanaka, and T. Okuda, Direct Evidence of Hidden Local Spin Polarization in a Centrosymmetric Superconductor $\mathrm{LaO}_{0.55} \mathrm{~F}_{0.45} \mathrm{BiS}_{2}$, Nat. Commun. 8, 1919 (2017).

[73] Y. J. Zhang, P. F. Liu, H. Y. Sun, S. X. Zhao, H. Xu, and Q. H. Liu, Symmetry-Assisted Protection and Compensation of Hidden Spin Polarization in Centrosymmetric Systems, Chin. Phys. Lett. 37, 087105 (2020).

[74] The commutation-anticommutation relation in Eq. (6) is true only in the sense of the result in Eq. (5b). In general, the commutation relation between a unitary operator and an antiunitary operator is not well defined, considering that the relation can be changed by a U(1) gauge.

[75] M. Sato, Y. Tanaka, K. Yada, and T. Yokoyama, Topology of Andreev Bound States with Flat Dispersion, Phys. Rev. B 83, 224511 (2011).

[76] D. F. Agterberg, T. Shishidou, J. O'Halloran, P. M. R. Brydon, and M. Weinert, Resilient Nodeless $d$-Wave Superconductivity in Monolayer FeSe, Phys. Rev. Lett. 119, 267001 (2017).

[77] F. Zhang, C. L. Kane, and E. J. Mele, Surface State Magnetization and Chiral Edge States on Topological Insulators, Phys. Rev. Lett. 110, 046404 (2013).

[78] W. A. Benalcazar, B. A. Bernevig, and T. L. Hughes, Quantized Electric Multipole Insulators, Science 357, 61 (2017).

[79] W. A. Benalcazar, B. A. Bernevig, and T. L. Hughes, Electric Multipole Moments, Topological Multipole Moment Pumping, and Chiral Hinge States in Crystalline Insulators, Phys. Rev. B 96, 245115 (2017).

[80] Z. D. Song, Z. Fang, and C. Fang, (d-2)-Dimensional Edge States of Rotation Symmetry Protected Topological States, Phys. Rev. Lett. 119, 246402 (2017).
[81] F. Schindler, A. M. Cook, M. G. Vergniory, Z. J. Wang, S. S. Parkin, B. A. Bernevig, and T. Neupert, HigherOrder Topological insulators, Sci. Adv. 4, eaat0346 (2018).

[82] J. Langbehn, Y. Peng, L. Trifunovic, F. von Oppen, and P. W. Brouwer, Reflection-Symmetric Second-Order Topological Insulators and Superconductors, Phys. Rev. Lett. 119, 246401 (2017).

[83] M. Geier, L. Trifunovic, M. Hoskam, and P. W. Brouwer, Second-Order Topological Insulators and Superconductors with an Order-Two Crystalline Symmetry, Phys. Rev. B 97, 205135 (2018).

[84] R. Jackiw and C. Rebbi, Solitons with Fermion Number 1/2, Phys. Rev. D 13, 3398 (1976).

[85] Z. B. Yan, F. Song, and Z. Wang, Majorana Corner Modes in a High-Temperature Platform, Phys. Rev. Lett. 121, 096803 (2018).

[86] Q. Y. Wang, C.-C. Liu, Y.-M. Lu, and F. Zhang, HighTemperature Majorana Corner States, Phys. Rev. Lett. 121, 186801 (2018).

[87] R.-X. Zhang, W. S. Cole, and S. D. Sarma, Helical Hinge Majorana Modes in Iron-Based Superconductors, Phys. Rev. Lett. 122, 187001 (2019).

[88] We list only the elemental phases here. The direct sum of the different elemental phases is also supported. For instance, we consider the direct sum of the phases in Figs. 5(b) and 5(d). In such a system, there are bulk nodes, quasi-1D Dirac cones spreading across the whole surface Brillouin zone along the $k_{z}$ direction, and flat Majorana pairs across the whole 1D Brillouin zone along the hinge.

[89] Y. Zhang, L. X. Yang, M. Xu, Z. R. Ye, F. Chen, C. He, H. Xu, J. Jiang, B. P. Xie, J. J. Ying, X. F. Wang, X. H. Chen, J. P. Hu, and D. L. Feng, Nodeless Superconducting Gap in $\mathrm{A}_{x} \mathrm{Fe}_{2} \mathrm{Se}_{2}(\mathrm{~A}=\mathrm{K}, \quad C s)$ Revealed by Angle-Resolved Photoemission Spectroscopy, Nat. Mater. 10, 273 (2011).

[90] T. Qian, X.-P. Wang, W.-C. Jin, P. Zhang, P. Richard, G. Xu, X. Dai, Z. Fang, J.-G. Guo, X.-L. Chen, and H. Ding, Absence of a Holelike Fermi Surface for the Iron-Based $\mathrm{K}_{0.8} \mathrm{Fe}_{1.7} \mathrm{Se}_{2}$ Superconductor Revealed by Angle-Resolved Photoemission Spectroscopy, Phys. Rev. Lett. 106, 187001 (2011).

[91] D. F Liu et al., Electronic Origin of High-Temperature Superconductivity in Single-Layer FeSe Superconductor, Nat. Commun. 3, 931 (2012).

[92] L. Zhao et al., Common Electronic Origin of Superconductivity in (Li, Fe)OHFeSe Bulk Superconductor and Single-Layer FeSe/ $\mathrm{SrTiO}_{3}$ Films, Nat. Commun. 7, 10608 (2016).

[93] P. C. Dai, Antiferromagnetic Order and Spin Dynamics in Iron-Based Superconductors, Rev. Mod. Phys. 87, 855 (2015).

[94] D. Huang and J. E. Hoffman, Monolayer FeSe on SrTiO3, Annu. Rev. Condens. Matter Phys. 8, 311 (2017).

[95] E. Dagotto, Colloquium: The Unexpected Properties of Alkali Metal Iron Selenide Superconductors, Rev. Mod. Phys. 85, 849 (2013).

[96] Q.-Y. Wang, Z. Li, W.-H. Zhang, Z.-C. Zhang, J.-S. Zhang, W. Li, H. Ding, Y.-B. Ou, P. Deng, K. Chang, J.Wen, C.-L. Song, K. He, J.-F. Jia, S.-H. Ji, Y.-Y. Wang, L.-L. Wang, X. Chen, X.-C. Ma, and Q.-K. Xue, Interface-Induced 
High-Temperature Superconductivity in Single Unit-Cell FeSe Films on $\mathrm{SrTiO}_{3}$, Chin. Phys. Lett. 29, 037402 (2012).

[97] C. Fang, Y.-L. Wu, R. Thomale, B. A. Bernevig, and J. P. $\mathrm{Hu}$, Robustness of $s$-Wave Pairing in Electron-Overdoped $\mathrm{A}_{1-y} \mathrm{Fe}_{2-x} \mathrm{Se}_{2}(\mathrm{~A}=\mathrm{K}$, Cs $)$, Phys. Rev. X 1, 011009 (2011).

[98] X. L. Lu, C. Fang, W.-F. Tsai, Y. J. Jiang, and J. P. Hu, s-Wave Superconductivity with Orbital-Dependent Sign Change in Checkerboard Models of Iron-Based Superconductors, Phys. Rev. B 85, 054505 (2012).

[99] Z. P. Yin, K. Haule, and G. Kotliar, Spin Dynamics and Orbital-Antiphase Pairing Symmetry in Iron-Based Superconductors, Nat. Phys. 10, 845 (2014).

[100] J. P. Hu, N. Hao, and X. X. Wu, Mechanism for Odd Parity Superconductivity in Iron-Based Superconductors, arXiv:1303.2624.

[101] N. Hao and J. P. Hu, Odd Parity Pairing and Nodeless Antiphase $s^{ \pm}$in Iron-Based Superconductors, Phys. Rev. B 89, 045144 (2014).

[102] Z. Y. Du, X. Yang, D. Altenfeld, Q. Q. Gu, H. Yang, I. Eremin, P. J. Hirschfeld, I. I. Mazin, H. Lin, X. Y. Zhu, and H.-H. Wen, Sign Reversal of the Order Parameter in $\left(\mathrm{Li}_{1-x} \mathrm{Fe}_{x}\right) \mathrm{OHFe}_{1-y} \mathrm{Zn}_{y} \mathrm{Se}$, Nat. Phys. 14, 134 (2018).

[103] Q. Fan, W. H. Zhang, X. Liu, Y. J. Yan, M. Q. Ren, R. Peng, H. C. Xu, B. P. Xie, J. P. Hu, T. Zhang, and D. L. Feng, Plain s-Wave Superconductivity in Single-Layer FeSe on $\mathrm{SrTiO}_{3}$ Probed by Scanning Tunnelling Microscopy, Nat. Phys. 11, 946 (2015).

[104] X. X. Wu, Y. Liang, H. Fan, and J. P. Hu, Nematic Orders and Nematicity-Driven Topological Phase Transition in FeSe, arXiv:1603.02055.

[105] An interesting comparison is the nodeless $d$-wave state. The $d$-wave state cannot support the full-gap topologically nontrivial superconductivity due to the following two facts. First, as pointed out in Ref. [76], the $d$-wave state can be nodeless only when the superconducting order dominates the spin-orbit coupling, namely, the weak spin-orbit coupling condition. Second, in the weak spin-orbit coupling condition, the system is topologically equivalent with spinless superconductors (the system remains fully gapped as the spin-orbit coupling $\lambda \rightarrow 0$ ). For a spinless spin-singlet superconductor, it is always topologically trivial. This is consistent with the common sense that, to realize topological superconductivity in spin-singlet superconductors, strong spinorbit coupling is necessary. More details are shown in Appendix G.

[106] The group structure in Eq. (2) is vital for topological superconductivity. Based on a query of all 230 space groups, we find that there are 61 centrosymmetric space groups which support similar group structure. We list these space groups in Appendix $\mathrm{H}$.

[107] J. Ahn and B.-J. Yang, Unconventional Majorana Fermions on the Surface of Topological Superconductors Protected by Rotational Symmetry, Phys. Rev. B 103, 184502 (2021).

[108] L. Fu and C. L. Kane, Topological Insulators with Inversion Symmetry, Phys. Rev. B 76, 045302 (2007).

[109] L. Fu and E. Berg, Odd-Parity Topological Superconductors: Theory and Application to $\mathrm{Cu}_{x} \mathrm{Bi}_{2} \mathrm{Se}_{3}$, Phys. Rev. Lett. 105, 097001 (2010).

[110] S. V. Borisenko, D. V. Evtushinsky, Z.-H. Liu, I. Morozov, R. Kappenberger, S. Wurmehl, B. Büchner, A. N. Yaresko, T. K. Kim, M. Hoesch, T. Wolf, and N. D. Zhigadlo, Direct Observation of Spin-Orbit Coupling in Iron-Based Superconductors, Nat. Phys. 12, 311 (2016). 